TRANSACTIONS OF THE

AMERICAN MATHEMATICAL SOCIETY

Volume 352, Number 12, Pages 5619-5666

S 0002-9947(00)02412-0

Article electronically published on July 13, 2000

\title{
POWER OPERATIONS IN ELLIPTIC COHOMOLOGY AND REPRESENTATIONS OF LOOP GROUPS
}

\author{
MATTHEW ANDO
}

\begin{abstract}
Part I of this paper describes power operations in elliptic cohomology in terms of isogenies of the underlying elliptic curve. Part II discusses a relationship between equivariant elliptic cohomology and representations of loop groups. Part III investigates the representation of theoretic considerations which give rise to the power operations discussed in Part I.
\end{abstract}

\section{INTRODUCTION}

0.1. Operations arising from isogenies. In this paper we shall consider unstable operations among complex-oriented cohomology theories which arise in the following situation. Suppose that $E$ is a commutative, 2-periodic ring theory with $E^{\text {odd }}(p t)=0$; we shall refer to such theories as homogeneous theories. A homogeneous theory is automatically complex-orientable, and $E^{0} \mathbb{C} P^{\infty}$ is the ring of formal functions on a formal group $G_{E}$ over $\pi_{0} E=E^{0}(p t)$ (see section 11). Suppose that $E$ and $F$ are two such theories, and one has the following algebraic data:

(i) a ring homomorphism $\pi_{0} F \stackrel{i}{\rightarrow} R$;

(ii) a ring homomorphism $\pi_{0} E \stackrel{\psi}{\longrightarrow} R$;

(iii) a homomorphism of formal groups $i^{*} G_{F} \stackrel{\pi}{\longrightarrow} \psi^{*} G_{E}$.

We denote such data $(i, \pi, \psi)$. If the theory $E$ is Landweber exact, the homomorphism $i$ is an isomorphism, and the map of formal groups $\pi$ is an isomorphism, then it is well-understood how these data give rise to a stable natural transformation

$$
P: E^{*}(-) \rightarrow F^{*}(-)
$$

of ring theories such that

$$
P(p t)=\psi
$$

and

$$
P\left(\mathbb{C} P^{\infty}\right)=\pi^{*}
$$

(see, for example, Mil89]; important issues concerning infinite complexes have recently been thoroughly investigated by [HS97]).

The first result of this paper is to relax the assumptions about $i$ and $\pi$ and still obtain an unstable operation.

Received by the editors October 23, 1995 and, in revised form, June 10, 1998.

1991 Mathematics Subject Classification. Primary 55N20, 55S25, 55N91, 22 E67.

Supported by the NSF.

(C)2000 American Mathematical Society 
Theorem A. If $E$ is Landweber exact, and there is an element $\lambda \in R$ which is not a zero-divisor such that $\pi$ becomes an isomorphism over $R\left[\frac{1}{\lambda}\right]$, then the data $(i, \pi, \psi)$ give rise to a natural transformation of ring-valued functors

$$
\underset{\psi}{R} E^{0}(-) \stackrel{\Psi}{\longrightarrow} \underset{i}{R} F^{0}(-)
$$

such that $\Psi\left(\mathbb{C} P^{\infty}\right)=\pi^{*}$.

In the statement we have used the notation

$$
R \hat{\otimes} F^{0}(X)=\lim _{Y \subset X} R \otimes F^{0}(Y)
$$

where $X$ is a CW complex and $Y$ ranges over the finite subcomplexes of $X$; see section 1 .

0.2. Operations in elliptic cohomology theories. Our main application is to elliptic cohomology theories. An elliptic cohomology theory is a homogeneous theory $E$, given with an elliptic curve $C$ over $\pi_{0} E$, and an isomorphism of formal groups $\widehat{C} \cong G_{E}$ (see section 21).

Suppose that $i: \pi_{0} E \rightarrow R$ is $\pi_{0} E$-algebra, $A$ is an abelian group, and

$$
A \stackrel{\phi}{\longrightarrow} i^{*} C
$$

is a Drinfel'd $A$-structure (see section 4); we abbreviate such data as $(i, \phi)$. Then the quotient $i^{*} C /[\phi]$ is an elliptic curve; we use the notation $\pi_{\phi}$ for the isogeny

$$
i^{*} C \stackrel{\pi_{\phi}}{\longrightarrow} i^{*} C /[\phi] \text {. }
$$

A factorization of $(i, \phi)$ is a pair $(\psi, g)$ consisting of

(i) a ring homomorphism $\pi_{0} E \stackrel{\psi}{\longrightarrow} R$,

(ii) an isomorphism of formal groups $i^{*} \widehat{C /[\phi]} \stackrel{g}{\cong} \psi^{*} \widehat{C}$.

From the factorization $(\psi, g)$ of the Drinfel'd $A$-structure $(i, \phi)$, one obtains a homomorphism of formal groups

$$
i^{*} \widehat{C} \stackrel{g \hat{\pi}_{\phi}}{\longrightarrow} \psi^{*} \widehat{C}
$$

Using the isomorphism $G_{E} \cong \widehat{C}$ and Theorem $\mathrm{A}$, one has

Corollary B. If $E$ is Landweber exact and the order of $A$ is not a zero-divisor in $R$, then a factorization $(\psi, g)$ of a Drinfel'd A-structure gives rise to an operation

$$
\underset{\psi}{\hat{\otimes}} E^{0}(-) \stackrel{\Psi}{\longrightarrow} \underset{i}{R} \hat{\otimes} E^{0}(-)
$$

whose effect on $\mathbb{C} P^{\infty}$ is $\left(g \hat{\pi}_{\phi}\right)^{*}$.

The proof of Theorem $\mathrm{A}$ and Corollary $\mathrm{B}$ will be given in section [5, It is well known how to produce Drinfel'd $A$-structures on an elliptic curve or a formal group; see section 4 A factorization $(\psi, g)$ can often be found when the elliptic curve $C$ over $\pi_{0} E$ or its formal group enjoys universally a property which is retained by the curve $i^{*} C /[\phi]$ over $R$. As an example, we show

Theorem C (Theorem 6.2). The elliptic cohomology theory associated to the Igusa quartic has canonical factorizations for Drinfel'd isogenies of odd order. 
Applying Corollary B yields the summands of the Hecke operator, given as integral unstable operations on the the elliptic cohomology constructed by Landweber, Ravenel, and Stong. As another example, we construct $K$-theoretic operations based on isogenies of the Tate elliptic curve.

0.3. Relationship to power operations. The proof of Theorem A uses standard constructions for Landweber-exact theories, Wilson's techniques for studying unstable operations [Wil82, and Ravenel and Wilson's calculation of Hopf rings for complex cobordism [RW77] as extended by [HH95] and HT98. The relationship with power operations appears when one tries to compute the effect of the operation $\Psi$ on $\mathbb{C} P^{\infty}$.

In the situation of Corollary $\mathrm{B}$, a coordinate $t$ on $\hat{C}$ gives two functions on $i^{*} \hat{C}$, namely the coordinate $i^{*} t$ and the function $\hat{\pi}_{\phi}^{*} g^{*} \psi^{*} t$. By Corollary B] one always has

$$
\Psi\left(\mathbb{C} P^{\infty}\right)\left(\psi^{*} t\right)=\hat{\pi}_{\phi}^{*} g^{*} \psi^{*} t,
$$

and one might wish to express this element in terms of the coordinate $i^{*} t$. We give three examples in section 6: the theory of Landweber, Ravenel and Stong, the elliptic cohomology based on the Tate curve, and the theory $E_{2}$.

In each case, there is a coordinate in terms of which the effect of the operation on $\mathbb{C} P^{\infty}$ is a norm for the isogeny $\hat{\pi}_{\phi}$. In the case of $E_{2}$, we explain how the norm is the shadow of the $H_{\infty}$ structure on $M U$. Because of the relationship to the $H_{\infty}$ structure on $M U$, we call the operations under study here power operations, even though we do not show that any elliptic cohomology theory is an $H_{\infty}$ ring spectrum in the sense of BMMS86].

The rest of the paper follows a lead suggested by analogy with $K$-theory. Power operations in $K$-theory have their origin in symmetries of the representation theory of compact Lie groups; indeed, any power operation $\Theta$ on $K$-theory extends to equivariant $K$-theory, and the effect of $\Theta$ on the representation ring $R(G)$ is precisely its effect on $K_{G}(p t)$. Part of the dream about elliptic cohomology has been that it possesses, for a compact Lie group $G$, an equivariant extension which is related to representations of the loop group

$$
L G=\mathrm{C}^{\infty}\left[S^{1}, G\right]
$$

of unbased smooth loops on $G$ Bry90, Seg88, just as equivariant $K$-theory is related to representations compact Lie groups. It seems natural to ask what the power operations in elliptic cohomology mean in the context of loop group representations.

Grojnowski and Ginzburg, Kapranov and Vasserot Gro94, GKV95] have developed a notion of $G$-equivariant elliptic cohomology in which the relationship with loop group representations takes a precise form; this relationship is the topic in the second part of this paper. It is independent from the first part, aside from a few well known facts about the Tate curve.

Let $G$ be a compact connected Lie group, $T$ a maximal torus, and $W$ its Weyl group. Let $\mathbb{T}$ be the circle group, so that

$$
\check{T} \stackrel{\text { def }}{=} \operatorname{Hom}[\mathbb{T}, T]
$$

is the group of cocharacters of $T$. Let $E$ be an elliptic cohomology theory, and $C$ the associated elliptic curve. Grojnowski Gro94 and Ginzburg, Kapranov and 
Vasserot GKV95] have explained that the fundamental object of study for $E_{G}$ is the abelian scheme

$$
\check{T} \underset{\mathbb{Z}}{\otimes} C
$$

together with its $W$-action. Grojnowski has constructed a complex equivariant theory based on this idea.

The first elliptic cohomology theory was discovered by Morava in 1973 (see [Mor89]): the elliptic curve is the Tate elliptic curve, Tate. Its formal group is the multiplicative group, so it is a form of $K$-theory. We call the theory $K_{\text {Tate }}$. Looijenga Loo76] shows that a simple, simply-connected Lie group $G$ determines canonically a $W$-equivariant line bundle $\mathcal{L}(G)$ over $\check{T} \otimes$ Tate; he also calculates its $W$-invariant sections. The main point of Part $\amalg$ is the following.

Theorem D. The character map establishes an isomorphism between the $\mathbb{Z}((q))$ module of representations of $L G$ of level $k$ and the $\mathbb{Z}((q))$-module of $W$-invariant global sections of $\mathcal{L}(G)^{k}$.

The precise statement of Theorem $\mathrm{D}$ is slightly more complicated, because of the difficulty in regarding the Tate curve as a quotient of the multiplicative group, and so of regarding theta functions as sections of line bundles. See Corollary 10.9 Theorem 10.10 and, in the toral case, Theorem 11.6.

The main ingredients in the proof of Theorem $\mathrm{D}$ are Kac's character formula and Looijenga's analysis [Lo076] of the line bundle $\mathcal{L}(G)$ and its sections. Indeed, Theorem $\mathrm{D}$ is essentially Theorem 13.3 of [Kac85]; although, there one works over $\mathbb{C}$, and the geometric picture is more difficult to discern. The result might have appeared in Looijenga's paper if Kac's character formula had not been so new at the time. As it happened, it fell to Grojnowski to assemble these results and connect them with elliptic cohomology; I am grateful to him for explaining these ideas to me. I hope that the present account will make these exciting results accessible to a wider audience.

In part III we work backwards from the effect of power operations on $K_{\text {Tate }}$ to identify operations on representations of loop groups. It turns out that the extra operations in elliptic cohomology (beyond those available in $K$-theory) reflect the interaction of the tensor powers and the circle group acting on loop groups by rotation; see Propositions 12.5, 12.7 and 13.3.

There is undoubtedly more to say on this interaction. If $G$ is simple, simply connected, and simply laced, then all the irreducible representations of $L G$ of level $n$ appear as summands in the "basic representation" $\mathcal{H}$ of level 1 , pulled back by the $n^{\text {th }}$ power map

$$
L G \stackrel{[n]}{\longrightarrow} L G
$$

on loops PS86 Proposition (9.3.9)]. (In fact, the same holds when $G$ is a unitary group [Fre82.) This pull-back may be viewed as the restriction to the subgroup of loops fixed by the action of the subgroup of order $n$ of the group of rotations acting on $L G$. Thus the symmetry which produces power operations in elliptic cohomology also generates the representation theory of certain loop groups, much the same as power operations in $K$-theory generate the representation theory of $U_{n}$ from its representation on $\mathbb{C}^{n}$. The decomposition into irreducible summands of $[n]^{*} \mathcal{H}$ has been studied extensively by Frenkel Fre82, Kac and Wakimoto [KW90. 
The relationship of their results to the present work remains unclear, and we hope to be able to return to it.

The idea of relating power operations to isogenies, originated in my dissertation. However, my understanding of these issues has been greatly influenced by Mike Hopkins and Neil Strickland, and Part \ reflects their tutelage. I learned a lot from some of Neil's manuscripts, particularly, Formal schemes and formal groups, and I thank him for making them available to me. The second part of this paper owes its existence to several long conversations with Ian Grojnowski and Mikhail Kapranov, who explained their papers to me and exhibited great patience with my plodding ways. I am grateful to Farshid Hajir and Amnon Neeman for conversations about theta functions, and to Haynes Miller and Jack Morava for conversations and encouragement about many parts of this project. Finally, I am very grateful to the referee, who made insightful suggestions, and pointed out important mistakes in earlier versions of this paper. Like the ones (s)he caught, the mistakes that remain are mine and mine alone.

\section{Part I. Power operations and elliptic cohomology}

\section{Complex-orientable cohomology theories}

We construct unstable ring operations, so if $E$ is a cohomology theory taking values in rings, it is convenient to use the notation $E^{*}(X)$ to refer to the unreduced cohomology of a space $X$; the reduced cohomology will be indicated by $\tilde{E}^{*}(X)$.

Definition 1.1. A ring-valued cohomology theory $E$ is complex orientable if there is a class $x \in \tilde{E}^{2}\left(\mathbb{C} P^{\infty}\right)$ such that

$$
i^{*} x=\Sigma^{2}(1) \in \tilde{E}^{2}\left(S^{2}\right) \cong E^{0}(p t),
$$

where

$$
S^{2} \stackrel{i}{\rightarrow} \mathbb{C} P^{\infty}
$$

is the inclusion of the bottom cell, and $\Sigma$ is the suspension isomorphism. A complex orientation is a choice of $x$.

Most of the examples we consider are two-periodic.

Definition 1.2. A ring theory $E$ is homogeneous if $E^{\text {odd }}(p t)=0$, and $E^{2}(p t)$ contains a unit of $E^{*}(p t)$.

A homogeneous theory is automatically complex-orientable, as we can easily see by calculating $E^{*} \mathbb{C} P^{\infty}$ using the Atiyah-Hirzebruch spectral sequence. In this case we abbreviate $E(X)$ for $E^{0}(X)$. We shall also occasionally write $\pi_{k} E$ for $\tilde{E}^{0}\left(S^{k}\right)=E^{-k}(p t)$ and even $E$ for $\pi_{0} E$.

Many of our constructions use the geometry of elliptic curves, which is easiest to describe in the language of schemes. If $E$ is a homogeneous ring theory, we use the notation $S_{E}$ for Spec $E(p t)$; thus $X \mapsto \operatorname{Spec} E(X)$ is a covariant functor from spaces to affine schemes over $S_{E}$.

In fact, we get slightly more, since maps between spaces preserve the augmentation ideal. Many spaces $X$ of particular interest in the study of complex-oriented theories have the property that $E(X)$ is complete with respect to the topology defined by its augmentation ideal. An opposite category of schemes which remembers this structure will ease the comparison to elliptic curves: we define the category of 
(pointed, affine) formal schemes over $S_{E}$ to be the opposite of the category of complete augmented $E$-algebras and continuous homomorphisms. If $R$ is a complete augmented $E$-algebra, we denote the corresponding formal scheme by $\operatorname{Spf} R$; and if $X$ is a formal scheme over $S_{E}$, we denote the corresponding algebra by $\mathcal{O}_{X}$. A much more thorough and detailed treatment of these ideas is given by Strickland Str98.

In fact, most of our examples will be produced from Landweber's exact functor theorem, so they really give well defined theories only on finite complexes. If $E$ is a cohomology theory on finite complexes, we define a functor on infinite complexes by writing

$$
\widehat{E}(X)=\lim _{Y \subset X} E(Y)
$$

where $Y$ runs over the finite subcomplexes. This is not a cohomology theory. However, $E$ does extend non-canonically to infinite complexes [Ada71. If $E^{\prime}$ is any such extension, then the natural map

$$
E^{\prime}(X) \rightarrow \widehat{E}(Y)
$$

is an isomorphism if, for example, $X$ has cells only in even degrees. This is the case for $X=\mathbb{C} P^{\infty}$ or $X=\Omega^{\infty} F$, where $F$ is $\mathcal{R W}$ (see section 3). Similarly, if $R$ is an $E$-algebra, then we write

$$
R \hat{\otimes}_{E} E(X)=\lim _{Y \subset X} R \underset{E}{\otimes} E(Y)
$$

where $Y$ runs over the finite subcomplexes.

A key point is that completion enters base change and products: the $E$-algebra $R$ defines a base change

$$
\text { Formal schemes over } S_{E} \stackrel{i^{*}}{\longrightarrow} \text { Formal schemes over } \operatorname{Spec} R
$$

given by

$$
\operatorname{Spf} A \mapsto \operatorname{Spf} R \hat{\otimes} A .
$$

Similarly, the product of two formal schemes is given by

$$
\operatorname{Spf} A \times \operatorname{Spf} B=\operatorname{Spf} A \hat{\otimes} B .
$$

In particular, if $E$ is a homogeneous, complex-orientable cohomology theory, and

$$
G_{E} \stackrel{\text { def }}{=} \operatorname{Spf} \widehat{E}\left(\mathbb{C} P^{\infty}\right),
$$

then Spf $\widehat{E}\left(\mathbb{C} P^{\infty} \times \mathbb{C} P^{\infty}\right) \cong G_{E} \times G_{E}$. The multiplication on $\mathbb{C} P^{\infty}$ induces a map of formal schemes

$$
G_{E} \times G_{E} \stackrel{m}{\longrightarrow} G_{E},
$$

which, together with the structural map and the identity section

$$
\begin{aligned}
& G_{E} \stackrel{p}{\rightarrow} S_{E} \\
& G_{E} \stackrel{e}{\longleftarrow} S_{E},
\end{aligned}
$$

gives $G_{E}$ the structure of a commutative group in the category of formal schemes over $E(p t)$. It has more structure, that of a "formal group". We describe it as follows, to ease the comparison with elliptic curves (see section 2). 
Geometrically, the augmentation ideal $\tilde{E}^{0}\left(\mathbb{C} P^{\infty}\right)$ is the ideal

$$
\mathcal{O}_{G_{E}}(-e) \stackrel{\text { def }}{=}\left\{f \in \mathcal{O}_{G_{E}} \mid e^{*} f=0\right\}=\tilde{E}^{0}\left(\mathbb{C} P^{\infty}\right)
$$

of functions which vanish at the identity, and we have a commutative diagram

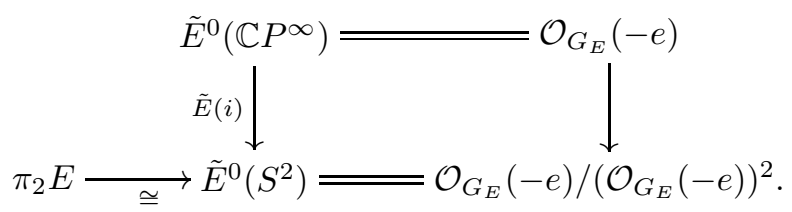

The free rank-one $E(p t)$-module $\mathcal{O}_{G_{E}}(-e) /\left(\mathcal{O}_{G_{E}}(-e)\right)^{2}$ is the module of sections of the sheaf

$$
\underline{\omega}_{E} \stackrel{\text { def }}{=} e^{*} \Omega_{G_{E} / E}^{1}
$$

of one-forms at the origin of $G_{E}$. Because of (1.3), we write

$$
t \mapsto e^{*} d t
$$

for the effect of the natural map

$$
\tilde{E}\left(\mathbb{C} P^{\infty}\right) \rightarrow \pi_{2} E .
$$

Definition 1.4. A coordinate on $G_{E}$ is a function

$$
t \in \mathcal{O}_{G_{E}}(-e)
$$

such that $e^{*} d t$ trivializes the sheaf $\underline{\omega}_{E}$.

A coordinate $t$ gives an isomorphism

$$
\mathcal{O}_{G_{E}} \cong E \llbracket t \rrbracket .
$$

Definition 1.5. A (commutative, one-dimensional) formal group over $R$ or $\operatorname{Spec} R$ is a commutative group in the category of formal schemes, isomorphic as a formal scheme to Spf $R \llbracket t \rrbracket$.

Remark 1.6. The multiplication on $G_{E}$ is determined by the element

$$
m^{*} t \in \mathcal{O}_{G_{E}} \hat{\otimes} \mathcal{O}_{G_{E}} \cong \pi_{0} E \llbracket s, t \rrbracket,
$$

which is a formal group law over $\pi_{0} E$, and for which we shall use the notation $\left(G_{E}, t\right)$.

Remark 1.7. It is important to be clear about the relationship between a coordinate (Definition 1.4) and an orientation (Definition 1.1). Let $t$ be a coordinate and let

$$
v=e^{*} d t \in \pi_{2} E
$$

be the associated one-form: then the element

$$
\frac{t}{v} \in \tilde{E}^{2}\left(\mathbb{C} P^{\infty}\right)
$$

is a complex orientation. On the other hand, if $x$ is an orientation and $v$ generates $\pi_{2} E$ as a $\pi_{0} E$ module, then $v x$ is a coordinate.

Lemma 1.8. A coordinate on $G_{E}$ is equivalent to a pair $(x, v)$ consisting of a complex orientation and a trivialization of the sheaf $\underline{\omega}_{E}$. 
Remark 1.9. The reader may complain that $K$-theory is the only classical example of a homogeneous theory. However, familiar complex-orientable examples give rise to homogeneous theories in a natural way: if $E$ is a cohomology theory taking its value in commutative rings with $\pi_{\text {odd }} E=0$, then

$$
E P^{*}(X) \stackrel{\text { def }}{=} \mathbb{Z}\left[u, u^{-1}\right] \underset{\mathbb{Z}}{\otimes} E^{*}(X)
$$

(with $|u|=2$ ) is a homogeneous theory (on finite complexes). This has the following geometric interpretation: let $C / R$ be a pointed smooth curve over a ring $R$, and let

$$
\operatorname{Spec} R \stackrel{e}{\rightarrow} C
$$

be its identity section. The sheaf

$$
\underline{\omega}_{C}=e^{*} \Omega_{C / R}^{1}
$$

is invertible and trivializable. The total space $T$ of the $\mathbb{G}_{m}$-torsor of trivializations of $\underline{\omega}$ is (non-canonically) Spec $R\left[u, u^{-1}\right]$. The $\mathbb{G}_{m}$ action grades the ring of functions on $T$; the degree of $u$ is 2 .

1.1. Example: $M P$. This example and the observation of its utility in the present context are due to Neil Strickland [Str98]. Start with the universal formal group law $(G, s)$ over Lazard's ring $L$. The differential $e^{*} d s$ trivializes the sheaf $\underline{\omega}_{G / L}$, so that $\left(G, s, u e^{*} d s\right)$ over $L\left[u, u^{-1}\right]$ is the universal triple (formal group, coordinate, nowhere-vanishing one-form). If $(G, s, \omega)$ is such a triple over a ring $R$, we shall use the notations

$$
\begin{gathered}
L \stackrel{(G, s)}{\longrightarrow} R \\
L\left[u, u^{-1}\right] \stackrel{(G, s, \omega)}{\longrightarrow} R
\end{gathered}
$$

for the resulting maps. We often omit the group $G$ from the notation when the context is sufficient.

$L\left[u, u^{-1}\right]$ is the coefficient ring of the cohomology theory represented by the spectrum

$$
M P \stackrel{\text { def }}{=} \bigvee_{k \in \mathbb{Z}} \Sigma^{2 k} M U .
$$

It comes equipped with a map of spectra

$$
s_{M P}: \mathbb{C} P^{\infty} \cong\left(\mathbb{C} P^{\infty}\right)^{L} \rightarrow \Sigma^{2} M U \rightarrow M P .
$$

Now suppose that $E$ is a homogeneous ring spectrum, and let $i: E \rightarrow R$ be an $E$-algebra. From a map of $E$-algebras

$$
E_{0} M P \stackrel{f}{\rightarrow} R
$$

we obtain an element $\left(E_{0} s_{M P}\right)^{*} f$ of

$$
\operatorname{Hom}_{E \text {-modules }}\left[\tilde{E}_{0}\left(\mathbb{C} P^{\infty}\right), R\right] \cong \mathcal{O}_{i^{*} G_{E}}(-e)
$$

which restricts to a generator of

$$
\operatorname{Hom}_{E \text {-modules }}\left[\tilde{E}_{0}\left(S^{2}\right), R\right],
$$

which is to say a trivialization of the sheaf $i^{*} \underline{\omega}_{E}$. In other words, we obtain a coordinate on $i^{*} G_{E}$.

Lemma 1.8 together with the standard argument for $M U$ shows that $M P$ enjoys the following universal property. 
Proposition 1.10. Let $E$ be a homogeneous, complex-orientable ring spectrum.

(i) If $i: E \rightarrow R$ is a ring homomorphism, then the natural map

$$
\operatorname{Alg}_{E}\left[E_{0} M P, R\right] \rightarrow \text { Coordinates on } i^{*} G_{E}
$$

is an isomorphism.

(ii) Taking $i$ to be the identity, the natural map

$$
\text { RingSpectra }[M P, E] \rightarrow \text { Coordinates on } G_{E}
$$

is an isomorphism.

Therefore, we use the notation $\left(G_{M P}, s_{M P}\right)$ for the universal formal group law.

\section{Elliptic COHOMOLOGY THEORIES}

If $C$ is an elliptic curve over $S$ with identity section

$$
S \stackrel{e}{\rightarrow} C,
$$

let $\mathcal{O}_{C, e}$ denote the stalk of germs of functions at the identity, and let $\widehat{\mathcal{O}}_{C, e}$ denote its completion at its augmentation ideal. Any function $t \in \mathcal{O}_{C, e}$ such that $e^{*} d t$ trivializes the sheaf $e^{*} \Omega_{C / S_{E}}$, determines an isomorphism

$$
\widehat{\mathcal{O}}_{C, e} \cong E \llbracket t \rrbracket .
$$

We use the notation $\widehat{C}$ for the formal scheme Spf $\widehat{\mathcal{O}}_{C, e}$. The group structure on $C$ induces a multiplication

$$
\widehat{C} \times \widehat{C} \rightarrow \widehat{C}
$$

so $\widehat{C}$ is a formal group over $S$.

Definition 2.1. An elliptic cohomology theory is a triple $(E, C, \kappa)$ consisting of

(i) a homogeneous theory $E$,

(ii) an elliptic curve $C \rightarrow S_{E}$,

(iii) an isomorphism $\kappa: G_{E} \cong \widehat{C}$ of formal groups over $S_{E}$.

When the isomorphism $\kappa$ is clear, we may omit it from the notation.

2.1. Example: Weierstrass. The closure of the cubic

$$
W_{0}: y^{2}=4 x^{3}-g_{2} x-g_{3} \stackrel{[x, y, 1]}{\longrightarrow} \mathbb{P}^{2}
$$

is an elliptic curve $W$ over $R=\mathbb{Z}[1 / 6]\left[g_{2}, g_{3}, \Delta, \Delta^{-1}\right] /\left(\Delta=g_{2}^{3}-27 g_{3}^{2}\right)$. Taking as origin the point $[0,1,0]$ at infinity, the function $t=x / y$ is a coordinate on $W$. The pair $\left(W, e^{*} d t\right)$ is the universal pair (elliptic curve, trivialization of sheaf of one-forms at origin) over $\mathbb{Z}[1 / 6]$-schemes [Del75]. The functor which associates to a $\mathbb{Z}[1 / 6]$ scheme the set of elliptic curves together with an ordered pair of trivializations of the sheaf of one-forms at the origin is represented by the ring $R\left[u, u^{-1}\right]$; the universal triple is $\left(W, e^{*} d t, u e^{*} d t\right)$.

In particular, the map

$$
L\left[u, u^{-1}\right] \stackrel{\left(\hat{W}, t, u e^{*} d t\right)}{\longrightarrow} R\left[u, u^{-1}\right]
$$

sends $u$ to $u$. Landweber's exact functor theorem shows that the functor

$$
X \mapsto M U^{*}(X) \underset{t / e^{*} d t}{\otimes} R\left[u, u^{-1}\right] \cong M P^{*}(X) \underset{\left(t, u e^{*} d t\right)}{\otimes} R\left[u, u^{-1}\right]
$$

is an elliptic cohomology theory on finite complexes. 
2.2. Example: Igusa. The closure $I^{\prime}$ of the quartic

$$
I_{0}: y^{2}=1-2 \delta x^{2}+\varepsilon x^{4}
$$

in $\mathbb{P}^{2}$ is singular at $[0,1,0]$, but its normalization, which is the closure in $\mathbb{P}^{3}$ of

$$
I_{0}: y^{2}=1-2 \delta x^{2}+\varepsilon x^{4} \stackrel{\left[x, x^{2}, y, 1\right]}{\longrightarrow} \mathbb{P}^{3},
$$

is an elliptic curve $I$ over $R=\mathbb{Z}[1 / 2]\left[\delta, \varepsilon, \Delta, \Delta^{-1}\right] /\left(\Delta=\varepsilon\left(\delta^{2}-\varepsilon\right)\right)$; the function $x$ is a coordinate at the identity $[0,0,0,1] \in I$. The map

$$
I \rightarrow I^{\prime}
$$

is an isomorphism away from $[0,1,0] \in I^{\prime}$, so $\hat{I}^{\prime}$ has canonically the structure of a formal group.

The Igusa quartic enjoys the following universal property. A triple $(C, \omega, P)$ consisting of an elliptic curve $C$ over a $\mathbb{Z}[1 / 2]$-scheme $S$, a trivialization $\omega$ of $\underline{\omega}$, and a point $P$ of exact order 2 , determines a map

$$
S \stackrel{\psi}{\longrightarrow} \operatorname{Spec} R
$$

together with a map

$$
C \stackrel{g}{\rightarrow} \psi^{*} I^{\prime}
$$

such that $\hat{g}$ is an isomorphism of formal groups. The function $g^{*} \psi^{*} x$ is the function on $C$ with divisor

$$
[2]^{-1}(e)-[2]^{-1}(P)
$$

normalized so that $\omega$ is $e^{*} g^{*} \psi^{*} d x$ Igu59].

The trivialization $e^{*} d x$ identifies the functor of trivializations of the sheaf $\underline{\omega}_{I}$ with $\operatorname{Spec} R\left[u, u^{-1}\right]$; the map

$$
L\left[u, u^{-1}\right] \stackrel{\left(x, u e^{*} d x\right)}{\longrightarrow} R\left[u, u^{-1}\right]
$$

sends $u$ to $u$. Once again, Landweber's exact functor theorem shows that

$$
X \mapsto M U^{*}(X) \underset{x / e^{*} d x}{\otimes} R\left[u, u^{-1}\right] \cong M P^{*}(X) \underset{\left(x, u e^{*} d x\right)}{\otimes} R\left[u, u^{-1}\right]
$$

is an elliptic cohomology theory on finite complexes; it is a 2-periodicization of the elliptic cohomology theory of [LRS95].

2.3. Example: Tate. Consider the power series

$$
\begin{aligned}
& s_{k}(q)=\sum_{n \geq 1} \frac{n^{k} q^{n}}{1-q^{n}}, \\
& a_{4}(q)=-5 s_{3}(q), \\
& a_{6}(q)=-\frac{5 s_{3}(q)+7 s_{5}(q)}{12} .
\end{aligned}
$$


Theorem 2.3. (i) The series $a_{6}(q)$ as well as $a_{4}(q)$ has coefficients in $\mathbb{Z} \llbracket q \rrbracket$.

(ii) The Weierstrass cubic

$$
y^{2}+x y=x^{3}+a_{4}(q) x+a_{6}(q)
$$

over $\mathbb{Z} \llbracket q \rrbracket$ has discriminant

$$
\Delta(q)=q \prod_{n \geq 1}\left(1-q^{n}\right)^{24} .
$$

Proof. See, for example, [Sil94, p. 410].

The Tate curve, Tate, is the curve over $\mathbb{Z} \llbracket q \rrbracket$ given by (2.4). It is a pointed curve of genus 1 over $\mathbb{Z} \llbracket q \rrbracket$. The point is located in the smooth part, which is a one-dimensional abelian group, and there is an isomorphism

$$
\widehat{\mathbb{G}}_{m} \cong \widehat{\text { Tate }}
$$

of formal groups (see DR73, VII, 1.16]).

The coordinate $x_{\text {Tate }}=x / y$ gives a trivialization $\omega_{\text {Tate }}$ of the sheaf $\underline{\omega}_{\text {Tate }}$, so the ring of functions on the $\mathbb{G}_{m}$-torsor of trivializations of $\underline{\omega}_{\text {Tate }}$ is canonically isomorphic to $\mathbb{Z} \llbracket q \rrbracket\left[u, u^{-1}\right]$.

It follows that the functor

$$
\begin{aligned}
X & \mapsto M U^{*}(X) \underset{x_{\text {Tate }} / e^{*} d x_{\text {Tate }}}{\otimes} \mathbb{Z} \llbracket q \rrbracket\left[u, u^{-1}\right] \\
& \cong M P^{*}(X) \underset{\left(x_{\text {Tate }}, u e^{*} d x_{\text {Tate }}\right)}{\otimes} \mathbb{Z} \llbracket q \rrbracket\left[u, u^{-1}\right]
\end{aligned}
$$

is a cohomology theory on finite complexes. Because its formal group is multiplicative, it is a form of $K$-theory [Mor89]; we call it $K_{\text {Tate }}$.

The formula (2.5) for the discriminant implies that over $\mathbb{Z}((q))$, the Tate curve is an elliptic curve. It is modeled on the multiplicative parameterization of elliptic curves over $\mathbb{C}$ : if $q$ is a complex number with $0<|q|<1$, then $\mathbb{C}^{\times} / q^{\mathbb{Z}}$ is an elliptic curve which fits into an exact sequence

$$
1 \rightarrow q^{\mathbb{Z}} \rightarrow \mathbb{C}^{\times} \rightarrow \mathbb{C}^{\times} / q^{\mathbb{Z}} \rightarrow 1
$$

we obtain in this way a family of elliptic curves

$$
\mathbb{C}^{\times} / q^{\mathbb{Z}} \rightarrow D^{\times}
$$

where

$$
D^{\times}(\mathbb{C})=\left\{q \in \mathbb{C}^{\times}|| q \mid<1\right\} .
$$

Indeed, for $q \in D^{\times}(\mathbb{C})$, the series $a_{4}(q)$ and $a_{6}(q)$ converge to complex numbers; let $E_{q}$ denote the resulting elliptic curve over $\mathbb{C}$. The series

$$
\begin{aligned}
& X(z, q)=\sum_{n \in \mathbb{Z}} \frac{q^{n} z}{\left(1-q^{n} z\right)^{2}}-2 s_{1}(q), \\
& Y(z, q)=\sum_{n \in \mathbb{Z}} \frac{\left(q^{n} u\right)^{2}}{\left(1-q^{n} u\right)}+s_{1}(q),
\end{aligned}
$$

define meromorphic functions of $z$ for $z \in \mathbb{C}^{\times}$. The function

$$
\begin{aligned}
\phi(z) & =[X(z, q): Y(z, q): 1], \quad z \notin q^{\mathbb{Z}}, \\
\phi\left(q^{r}\right) & =e=[0: 1: 0]
\end{aligned}
$$


defines an analytic isomorphism

$$
\mathbb{C}^{\times} / q^{\mathbb{Z}} \cong E_{q}
$$

see [Sil94 p. 410].

Alas, $q \in D^{\times}(\mathbb{C})$ is not a $\mathbb{C}$-valued point of $\operatorname{Spec} \mathbb{Z}((q))$. However, if $F$ is a non-archimedean field, let

$$
D^{\times}(F)=\left\{q \in F^{\times}|| q \mid<1\right\} .
$$

The function

$$
\begin{aligned}
\operatorname{Hom}^{\text {cts }}[\mathbb{Z}((q)), F] & \rightarrow D^{\times}(F) \\
g & \mapsto g(q)
\end{aligned}
$$

is an isomorphism of sets.

Theorem 2.7. For any continuous homomorphism

$$
\mathbb{Z}((q)) \stackrel{g}{\rightarrow} F,
$$

the procedure above gives an isomorphism of groups

$$
F^{\times} / g(q)^{\mathbb{Z}} \cong g^{*} \operatorname{Tate}(F) .
$$

Proof. See, for example, [Sil94, p. 423].

Using the function $z=1-x_{\text {Tate }}$, we define another coordinate $\theta(z, q)$ on the formal group Tate by the formula

$$
\theta(z, q)=(1-z) \prod_{k \geq 1}\left(1-q^{k} z\right)\left(1-q^{k} z^{-1}\right) .
$$

It does not extend to a function on the Tate curve. Instead, it is a variant of the Weierstrass $\sigma$ function: if $F$ is either $\mathbb{C}$ or a $p$-adic field, then for $q \in D^{\times}(F), \theta$ defines a function on $F^{\times}$, which has zeroes of first order at the points $q^{\mathbb{Z}}$ and which satisfies the equation

$$
\theta(q z, q)=-\frac{1}{z} \theta(z, q)
$$

Its relevance here is that $\theta(-z, q)$ is the character of the basic representation of the loop group of a circle; see section 11

We recall a model for the torsion of the Tate curve from [KM85, §8]. Let $A=$ $\mathbb{Z}\left[q, q^{-1}\right]$, and let $\tilde{T}[N]$ be the $A$-scheme which is the disjoint union

$$
\tilde{T}[N]=\bigcup_{i \in \mathbb{Z}} T_{i}[N]
$$

Here $T_{i}[N]$ is the scheme

$$
T_{i}[N]=\operatorname{Spec}\left(A[t] /\left(t^{N}-q^{i}\right)\right) .
$$

If $R$ is an $A$-algebra, then

$$
T_{i}[N](R)=\left\{r \in R^{\times} \mid r^{N}=q^{i}\right\} .
$$

The scheme $\tilde{T}[N]$ is a group scheme via the multiplication

$$
\begin{aligned}
T_{i}[N] \times T_{j}[N] & \rightarrow T_{i+j}[N] \\
(r, s) & \mapsto r s .
\end{aligned}
$$


The constant group $\mathbb{Z}$ is a subgroup of $\tilde{T}[N]$ by

$$
k \mapsto q^{k} \in \tilde{T}_{N k}[N] ;
$$

we denote this subgroup $q^{\mathbb{Z}}$. Let $T[N]$ be the quotient $\tilde{T}[N] / q^{\mathbb{Z}}$; as a scheme it is isomorphic to the subscheme

$$
\bigcup_{0 \leq i \leq N-1} T_{i}[N]
$$

of $\tilde{T}[N]$. As a group it fits into a short exact sequence of group schemes

$$
\begin{aligned}
\mu[N] \cong T_{0}[N] \hookrightarrow T[N] & \rightarrow \mathbb{Z}\left[\frac{1}{N}\right] / \mathbb{Z} \\
T_{i} & \mapsto \frac{i}{N} \quad \bmod \mathbb{Z} .
\end{aligned}
$$

Here $\mu[N]$ denotes the group scheme of $N^{\text {th }}$ roots of unity.

The maps

$$
\begin{aligned}
T_{i}[N] & \rightarrow T_{i k}[N k] \\
r & \mapsto r
\end{aligned}
$$

for various $i, k$, and $N$ induce homomorphisms

$$
T[N] \rightarrow T[N k]
$$

Let $T$ be the resulting ind-groupscheme; it fits into a short exact sequence

$$
1 \rightarrow \mu \rightarrow T \rightarrow \mathbb{Q} / \mathbb{Z} \rightarrow 0
$$

of ind-groupschemes.

Theorem 2.9. Over $\mathbb{Z}((q))$ there is a canonical isomorphism of ind-groupschemes

$$
\text { Tate }_{\text {tors }} \cong T
$$

which is compatible with the isomorphisms (2.6) and

$$
\mu \cong T_{0} \text {. }
$$

Proof. See [KM85, §8].

If $R$ is a complete topological ring, then a continuous homomorphism

$$
\mathbb{Z}((q)) \stackrel{f}{\rightarrow} R
$$

is determined by $f(q)$, and we often follow the convention of writing Tate $(f(q))$ and $T(f(q))[N]$ for $f^{*}$ Tate and $f^{*} T[N]$, etc.

2.4. Example: $E_{2}$. Let $C_{0}$ be a supersingular elliptic curve over a perfect field $k$ of characteristic $p>0$, and let $E\left(C_{0}\right)$ be the Lubin-Tate ring of deformations of its formal group $\widehat{C_{0}}$. The Serre-Tate Theorem (see, for example, Kat81]) gives a canonical lift $C$ of $C_{0}$ to $E\left(C_{0}\right)$, such that $\widehat{C}$ is the universal deformation of $\widehat{C_{0}}$.

A choice of coordinate $t$ on $\widehat{C}$ gives ring homomorphism

$$
\pi_{0} M P \stackrel{(\widehat{C}, t)}{\longrightarrow} E\left(C_{0}\right)
$$

which satisfies the hypotheses of Landweber's exact functor theorem, so the functor

$$
E\left(C_{0}\right)^{*}(-) \stackrel{\text { def }}{=} M P^{*}(-) \underset{(\widetilde{C}, t)}{\otimes} E\left(C_{0}\right)
$$


is a cohomology theory on finite complexes. Indeed a choice of $p$-typical coordinate $t$ determines an isomorphism

$$
E\left(C_{0}\right)^{*}(p t) \cong \mathbb{W} k \llbracket u_{1} \rrbracket\left[u, u^{-1}\right],
$$

exhibiting $E\left(C_{0}\right)$ as a cousin of the "completed" 2-periodic $E_{2}$ of [JW73, BW89]. More generally, if $G_{0}$ is a formal group over $k$ of height $n$, let $E\left(G_{0}\right)$ be the LubinTate ring of deformations of $G_{0}$. A choice of coordinate $t$ on the universal lift $G$ defines a cohomology theory $E\left(G_{0}\right)$, which is a cousin of the completed 2-periodic $E_{n}$.

\section{Uniqueness AND INTEGRALITy USING Hopf RINGS}

The preceding section dealt with cohomology theories defined on finite CW complexes. In this and later sections we shall discuss unstable natural transformations between such theories. Since the operations we construct are multiplicative, we treat these cohomology theories as functors from finite complexes to rings. Such theories and operations can be extended to infinite complexes, although in general not canonically. However, with the Hopf ring techniques initiated by [RW77, Wil82, it is possible to remove much of the ambiguity with regard to infinite complexes, at least unstably. The following propositions cover the situations which arise in this paper.

Suppose that $E$ and $F$ are homogeneous theories, and that $F_{0} \Omega^{\infty} E$ has a Künneth isomorphism (the space $\Omega^{\infty} E$ appears not to be uniquely defined; Corollary 3.3 shows that this is not the case). Choosing coordinates $s \in E^{0} \mathbb{C} P^{\infty}$ and $t \in F^{0} \mathbb{C} P^{\infty}$ gives classes

$$
\beta_{i} \in F_{0} \mathbb{C} P^{\infty}
$$

for $i \geq 1$ and a map

$$
\mathbb{C} P^{\infty} \stackrel{s}{\rightarrow} \Omega^{\infty} E .
$$

We then have classes $[a]$ and $b_{i}$ in $F_{0} \Omega^{\infty} E$ for $a \in \pi_{0} E$ and $i \geq 1$, defined by

$$
\begin{aligned}
\pi_{0} \Omega^{\infty} E & \rightarrow F_{0} \Omega^{\infty} E \\
a & \mapsto[a], \\
F_{0} \mathbb{C} P^{\infty} & \stackrel{F_{0} s}{\longrightarrow} F_{0} \Omega^{\infty} E \\
\beta_{i} & \mapsto b_{i}
\end{aligned}
$$

(the map (3.1a) is the Hurewicz homomorphism for $F$ ).

The methods of [Wil82] apply when $E_{0} \Omega^{\infty} F$ is the quotient of the free Hopf ring on the generators (3.1) by the relation of Theorem 3.8 in [RW77] (in fact, we shall use here only the fact that the classes (3.1) generate the Hopf ring). If $E$ is such that $F_{0} \Omega^{\infty} E$ is of this form for all homogeneous $F$, then we say that $E$ is $\mathcal{R W}$. Hopkins and Hunton [HH95] show that $E$ is $\mathcal{R} \mathcal{W}$ if it is Landweber exact, and $\pi_{0} E$ is countable over a subring of $\mathbb{Q}$. More recently, Goerss and independently Hunton and Turner [HT98] have removed the hypothesis of countability.

As an example of the utility of this description, we first observe the following uniqueness. 
Proposition 3.2. Let $E$ and $F$ be two homogeneous, complex-orientable ring spectra, and suppose that $E$ is $\mathcal{R} \mathcal{W}$. Suppose that a and $b$ are two maps

$$
\Omega^{\infty} E \stackrel{a, b}{\longrightarrow} \Omega^{\infty} F
$$

such that $a \circ f$ and $b \circ f$ are homotopic for any map

$$
X \stackrel{f}{\rightarrow} \Omega^{\infty} E
$$

out of a finite complex $X$. Then $a$ and $b$ are homotopic.

Proof. If $E$ is $\mathcal{R W}$, then there are isomorphisms

$$
\begin{aligned}
{\left[\Omega^{\infty} E, \Omega^{\infty} F\right] } & =F^{0}\left(\Omega^{\infty} E\right) \\
& \cong \operatorname{Hom}_{F}\left[F_{0} \Omega^{\infty} E, F\right] .
\end{aligned}
$$

Moreover, $F_{0} \Omega^{\infty} E$ is spanned as an $F$-module by classes which are in the $F$ homology image of maps to $\Omega^{\infty} E$ from finite products of spheres and finite complex projective spaces.

Corollary 3.3. For example, the proposition shows that an isomorphism

$$
E^{*}(-) \stackrel{c(-)}{\longrightarrow} F^{*}(-)
$$

of functors on the category of finite complexes gives rise to a well defined homotopy equivalence

$$
\Omega^{\infty} E \rightarrow \Omega^{\infty} F
$$

3.1. Integrality of unstable operations. We recall and slightly generalize Wilson's method in [Wil82, §11] for identifying integral unstable operations. Suppose that $E$ and $F$ are homogeneous theories, $E$ is $\mathcal{R} \mathcal{W}$, and $S$ is an $F$-algebra. The generalization involves the study of natural transformations of ring-valued functors

$$
E(-) \rightarrow S \hat{\otimes} F(-) .
$$

Of course $S \hat{\otimes} F$ is not, in general, a cohomology theory, but it is still true that an additive natural transformation

$$
E(-) \rightarrow S \hat{\otimes} F(-)
$$

is given by a primitive element $g \in P S \hat{\otimes} F\left(\Omega^{\infty} E\right)$.

The assumption that $E$ is $\mathcal{R W}$ implies that $\Omega^{\infty} E$ is weakly equivalent to a $\mathrm{CW}$ complex $C$ with cells concentrated in even degrees. For any finite subcomplex $Y$ of $C$ the natural map

$$
S \otimes F^{0} Y \rightarrow \operatorname{Hom}_{F}\left[F_{0} Y, F\right] \otimes S
$$

is an isomorphism. Using these $F_{0} Y$ to filter $F_{0} \Omega^{\infty} E$, it follows that there are isomorphisms

$$
\begin{aligned}
S \hat{\otimes} F^{0} \Omega^{\infty} E & \cong \operatorname{Hom}_{F}\left[F_{0} \Omega^{\infty} E, F\right] \hat{\otimes} S, \\
P S \hat{\otimes} F^{0} \Omega^{\infty} E & \cong \operatorname{Hom}_{F}\left[Q F_{0} \Omega^{\infty} E, F\right] \hat{\otimes} S,
\end{aligned}
$$

where $Q F_{0} \Omega^{\infty} E$ denotes the $F$-module of indecomposables of $F_{0} \Omega^{\infty} E$. 
Now suppose that $i: F \rightarrow R$ is an $F$-algebra, and $\lambda \in R$ is not a zero-divisor, so that $R \rightarrow R\left[\frac{1}{\lambda}\right]$ is injective. Let us use the notations

$$
\begin{aligned}
& R(-)=R \underset{i}{\hat{\otimes}} F(-), \\
& T(-)=R\left[\frac{1}{\lambda}\right] \underset{i}{\hat{\otimes}} F(-)
\end{aligned}
$$

for the indicated ring-valued functors.

Given a natural transformation, we suppose

$$
E(-) \stackrel{g}{\rightarrow} T(-)
$$

and so a well defined element $\hat{g}$ of

$$
P T^{0} \Omega^{\infty} E \cong \operatorname{Hom}_{F}\left[Q F_{0} \Omega^{\infty} E, F\right] \hat{\otimes} R\left[\frac{1}{\lambda}\right] .
$$

Proposition 3.6 (Wilson). The operation g refines to a natural transformation

$$
E(-) \stackrel{g}{\rightarrow} R(-)
$$

if and only if $g(p t)$ factors through $R(p t)$ and $g\left(\mathbb{C} P^{\infty}\right)$ factors through $R\left(\mathbb{C} P^{\infty}\right)$.

Proof. The only if direction is obvious. So suppose that $g(p t)$ and $g\left(\mathbb{C} P^{\infty}\right)$ factor through $R(-)$. The hypotheses give that in the diagram

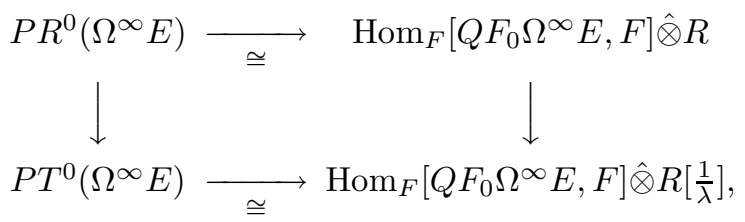

the horizontal arrows are isomorphisms and the vertical arrows are monomorphisms. Choose coordinates $s$ and $t$ on $G_{E}$ and $G_{F}$, and let $[a]$ and $b_{i}$ be defined as (3.1). The $F$-module $Q F_{0} \Omega^{\infty} E$ is spanned as an $F$-module by the image from $F_{0} \Omega^{\infty} E$ of elements of the form

$$
x=[a] \circ b_{1}^{\circ i_{1}} \circ b_{2}^{\circ i_{2}} \circ \cdots \circ b_{r}^{\circ i_{r}} .
$$

If

$$
g\left(\mathbb{C} P^{\infty}\right)(s)=\sum_{i \geq 1} u_{i} t^{i},
$$

then it is not hard to check that

$$
\begin{aligned}
\hat{g}([a]) & =g(p t)(a), \\
\hat{g}\left(b_{k}\right) & =u_{k}, \\
\hat{g}(x) & =(g(p t)(a)) \prod_{k=1}^{r} u_{k}^{i_{k}} .
\end{aligned}
$$

It follows that $g$ induces an operation

$$
E(-) \rightarrow \underset{i}{R} \hat{\otimes} F(-)
$$

as promised.

The same methods give the following result.

Proposition 3.7. If $E$ is $\mathcal{R} \mathcal{W}$, then two ring operations from $E^{0}(-)$ to $S \hat{\otimes} F^{0}(-)$ coincide if they coincide on a point and $\mathbb{C} P^{\infty}$. 


\section{DRINFEL'D ISOGENIES}

4.1. Level structures and isogenies. It will be helpful to recall some facts about level structures and isogenies of formal groups and elliptic curves. We start with a formal group or elliptic curve $C$ over a scheme $S$, and an abelian group $A$. If $C$ is a formal group, then we suppose that $S$ is the formal spectrum of a complete local ring.

Definition 4.1 (See KM85]). A Drinfel'd A structure on $C$

$$
A \stackrel{\phi}{\rightarrow} C
$$

is a homomorphism of groups

$$
A \stackrel{\phi}{\longrightarrow} C(S)
$$

such that the Cartier divisor

$$
[\phi] \stackrel{\text { def }}{=} \sum_{a \in A}[\phi(a)]
$$

is a subgroup scheme of $C$.

If $\phi: A \rightarrow C$ is a Drinfel'd $A$ structure, then, in particular, the subgroup-scheme $[\phi]$ is finite and flat over $S$. In that case, the quotient of $C$ by $[\phi]$ is a formal group or elliptic curve, according to the type of $C$ (see [Mum70, §12], [DG70, Ch. III, §2], [Lub67], and [Str97]).

Definition 4.2. A Drinfel'd isogeny is a sequence

$$
A \stackrel{\phi}{\rightarrow} C \stackrel{\pi}{\longrightarrow} D
$$

consisting of an isogeny $\pi$ and a Drinfel'd level structure $\phi$ such that

$$
[\phi]=\operatorname{Ker} \pi \text {. }
$$

4.2. Cohomology theories with level structures. It is easy to produce Drinfel'd isogenies on elliptic curves or formal groups.

Proposition 4.3. If $C$ is an elliptic curve or formal group over a ring $E$, and $A$ is a finite abelian group, then there is a universal pair $(i, \phi)$ consisting of an E-algebra

$$
E \stackrel{i}{\rightarrow} R
$$

(in case $C$ is a formal group, $i$ is a continuous map of complete local rings) and a Drinfel'd level structure

$$
A \stackrel{\phi}{\longrightarrow} i^{*} C
$$

The homomorphism $i$ is flat.

Proof. If $C$ is an elliptic curve and $A$ is cyclic or isomorphic to $(\mathbb{Z} / N)^{2}$, this is a result of Dri73 and KM85, Theorem 5.1.1]. For general $A$ and $C$ a formal group, see [Str97]. The case of a formal group is in turn the difficult input (axiom 4B) of Theorem 5.2.1 in KM85, which gives the case of general $A$ and an elliptic curve C. 
In particular, if $(E, C, \kappa)$ is an elliptic cohomology theory, then the functor

$$
X \mapsto F^{*}(X)=R \underset{i}{\otimes} E^{*}(X)
$$

is a cohomology theory on finite complexes, equipped with a map

$$
E(-) \stackrel{i}{\rightarrow} F(-)
$$

of ring theories.

\subsection{The norm of a coordinate. Let}

$$
A \stackrel{\phi}{\rightarrow} C \stackrel{\pi}{\rightarrow} D
$$

be a Drinfel'd isogeny over $S$. Let $t$ be a function on $C$ for which $t(\phi(a)) \neq \infty$ for $a \in A$, and define

$$
N_{\phi} t=\prod_{a \in A} \tau_{\phi(a)}^{*} t,
$$

where $\tau_{\phi(a)}: C \rightarrow C$ is translated by $\phi(a)$. The standard construction of the quotient $D$ ([Mum70, §12] and [DG70, Ch. III, §2]), together with Proposition 1.10.6 in KM85, shows that $N_{\phi} t$ descends to a function $\tilde{N}_{\phi} t$ on $D$. Now suppose, in addition, that $t$ is a coordinate on $C$. In section 6.1 it will be useful to know when $\tilde{N}_{\phi} t$ is a coordinate on $D$.

Proposition 4.5. $\tilde{N}_{\phi} t$ is a coordinate on $D$ if and only if the following condition is satisfied at each geometric point $x$ of $S$ : for all $a \in A$, we have $t_{x}(\phi(a))=0$ if and only if $\phi(a)=e_{x}$.

Proof. It suffices to check that $\tilde{N}_{\phi} t$ is a coordinate on the fiber at each geometric point of $S$, so by base change we are reduced to the case that $S$ is the spectrum of an algebraically closed field $k$.

If the characteristic of $k$ is zero, then $\pi$ is unramified so

$$
C \stackrel{\pi}{\rightarrow} D
$$

is étale. Then $\tilde{N}_{\phi} t$ is a coordinate on $D$ if and only if $N_{\phi} t$ is a coordinate on $C$. The equation

$$
e^{*} d N_{\phi} t=\lambda e^{*} d t
$$

where

$$
\lambda=\prod_{0 \neq a \in A} t(\phi(a))
$$

shows that $\tilde{N}_{\phi} t$ is a coordinate if and only if $t(\phi(a)) \neq 0$ for $0 \neq a \in A$.

If the characteristic of $k$ is $p>0$, then $\pi$ ramifies, say to degree $q=p^{r}$. In this case $\pi$ factors as

$$
C \stackrel{F r}{\longrightarrow} C^{(q)} \stackrel{u}{\longrightarrow} D
$$

where $F r$ is the $q$-power Frobenius and $u$ is étale [Sil86, II, $\S 2] . \tilde{N}_{\phi} t$ is a coordinate on $D$ if and only if $u^{*} \tilde{N}_{\phi} t$ is a coordinate on $C^{(q)}$. Let $A_{f} \subset A$ be the subgroup

$$
A_{f}=\{a \in A \mid \phi(a)=e\} .
$$

Let $A_{e} \rightarrow A$ be a splitting of $A \rightarrow A / A_{f}$; and write $\gamma$ for the composition

$$
A_{e} \stackrel{\phi}{\rightarrow} C \rightarrow C^{(q)} \text {. }
$$


Then

$$
A_{e} \stackrel{\gamma}{\rightarrow} C^{(q)} \stackrel{u}{\longrightarrow} D
$$

is a Drinfel'd isogeny. Let $z$ be the coordinate on $C^{(q)}$ such that $(F r)^{*} z=t^{q}$. We have

$$
\begin{aligned}
\pi^{*} \tilde{N}_{\phi} t & =\prod_{a \in A} \tau_{\phi(a)}^{*} t \\
& =\prod_{a \in A_{e}} \prod_{b \in A_{f}} \tau_{\phi(a)}^{*} \tau_{\phi(b)}^{*} t \\
& =\prod_{a \in A_{e}} \tau_{\phi(a)}^{*}\left(\prod_{b \in A_{f}} \tau_{\phi(b)}^{*} t\right) \\
& =\prod_{a \in A_{e}} \tau_{\phi(a)}^{*}\left(t^{q}\right) \\
& =F^{*}\left(\prod_{a \in A_{e}} \tau_{\gamma(a)} z\right),
\end{aligned}
$$

so

$$
u^{*} \tilde{N}_{\phi} t=N_{\gamma} z .
$$

For $\gamma$ (4.6) shows that $\tilde{N}_{\phi} t$ is a coordinate on $D$ if and only if $t(\phi(a)) \neq 0$ for $0 \neq a \in A_{e}$.

\section{UNSTABLE OPERATIONS FROM HOMOMORPHISMS OF FORMAL GROUPS}

Suppose that $F$ is a homogeneous theory; $i: \pi_{0} F \rightarrow R$ is a $\pi_{0} F$-algebra; and

$$
i^{*} G_{F} \stackrel{\pi}{\longrightarrow} H
$$

is a homomorphism of formal groups, which becomes an isomorphism after inverting an element $\lambda \in R$ which is not a zero-divisor. Let $u$ be a coordinate on $H$.

Proposition 5.1. There is an unstable natural transformation

$$
M P^{0}(-) \stackrel{Q(-)}{\longrightarrow} \underset{i}{\otimes} F^{0}(-)
$$

of ring-valued functors, such that $Q(p t)$ classifies the formal group law $(H, u)$, and

$$
Q\left(\mathbb{C} P^{\infty}\right)\left(s_{M P}\right)=\pi^{*} u \in \underset{i}{R} \hat{\otimes} F^{0}\left(\mathbb{C} P^{\infty}\right) .
$$

Proof. The coordinate $u$ gives a function $\pi^{*} u$ on $i^{*} G_{E}$, which is a coordinate over $R\left[\frac{1}{\lambda}\right]$. By Proposition 1.10 it is classified by a ring homomorphism

$$
f \in \operatorname{Alg}_{F}\left[F_{0} M P, R\left[\frac{1}{\lambda}\right]\right] .
$$

Using the fact that $M P$ is $\mathcal{R W}$ and the isomorphisms (3.5), we obtain from $f$ an element $\widehat{Q}$ of

$$
\begin{aligned}
\operatorname{Hom}_{F}\left[Q F_{0} \Omega^{\infty} M P, R\left[\frac{1}{\lambda}\right]\right] & \cong \operatorname{Hom}_{F}\left[Q F_{0} \Omega^{\infty} M P, F\right] \underset{i}{\hat{\otimes}} R\left[\frac{1}{\lambda}\right] \\
& \cong P R\left[\frac{1}{\lambda}\right] \underset{i}{\hat{\otimes}} F^{0}\left(\Omega^{\infty} M P\right) .
\end{aligned}
$$


Let $Q$ denote the resulting natural transformation (of ring-valued functors)

$$
M P^{0}(-) \rightarrow R\left[\frac{1}{\lambda}\right] \underset{i}{\hat{\otimes}} F^{0}(-) .
$$

By construction we have $Q\left(\mathbb{C} P^{\infty}\right)\left(s_{M P}\right)=\pi^{*} u$, so $Q(p t)$ classifies the formal group law $\left(i^{*} G_{F}, \pi^{*} u\right)=(H, u)$. Proposition 3.6 now implies the result.

5.1. Proof of Theorem $\mathbf{A}$. We are now prepared to give a proof of Theorem $A$ Thus suppose that $E$ and $F$ are homogeneous theories; $E$ is Landweber-exact;

$$
\begin{aligned}
& \pi_{0} F \stackrel{i}{\rightarrow} R \\
& \pi_{0} E \stackrel{\psi}{\longrightarrow} R
\end{aligned}
$$

are ring homomorphisms, and that $\pi$ is a homomorphism of formal groups

$$
i^{*} G_{F} \stackrel{\pi}{\longrightarrow} \psi^{*} G_{E}
$$

which becomes an isomorphism when $\lambda \in R$ is inverted. The element $\lambda$ is further required not to be a zero-divisor.

Choose a coordinate $u$ on $G_{E}$. By Proposition 1.10, this gives a ring operation

$$
M P^{0}(-) \stackrel{t}{\rightarrow} E^{0}(-)
$$

such that $t(p t)$ classifies the group law $\left(G_{E}, u\right)$. By Proposition 5.1 it also gives a ring operation

$$
M P^{0}(-) \stackrel{Q}{\longrightarrow} R \underset{i}{\otimes} F^{0}(-)
$$

such that $Q(p t)$ classifies $\left(G_{E}, \psi^{*} u\right)$; in other words,

$$
Q(p t)=\psi \circ t(p t)
$$

It follows that the diagram

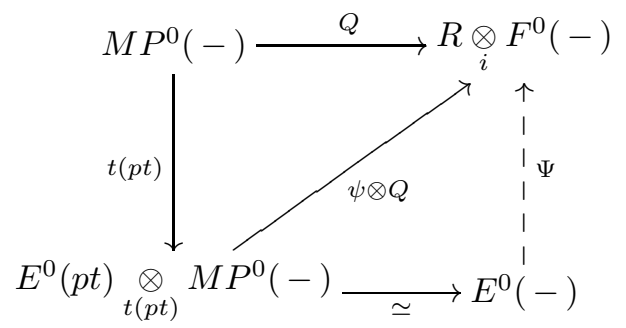

commutes; since $E$ is Landweber-exact, the natural map

$$
E^{0}(p t) \underset{t(p t)}{\otimes} M P^{0}(-) \stackrel{t}{\rightarrow} E^{0}(-)
$$

is an isomorphism, which fills in the dotted arrow. By construction, the effect of $\Psi$ on $u \in \underset{\psi}{R} \hat{\otimes} E^{0}\left(\mathbb{C} P^{\infty}\right)$ is given by

$$
\Psi\left(\mathbb{C} P^{\infty}\right)(u)=Q\left(\mathbb{C} P^{\infty}\right)\left(s_{M P}\right)=\pi^{*} u,
$$

So

$$
\Psi\left(\mathbb{C} P^{\infty}\right)=\pi^{*}
$$

Equation (5.2) shows that the operation $\Psi$ is independent of the choice of coordinate $u$. More precisely, let $s$ be another coordinate on $\hat{C}$, and denote by $\Psi_{s}$ and 
$\Psi_{t}$ the two elements of $R \underset{i}{\hat{\otimes}} F^{0}\left(\Omega^{\infty} E\right)$ produced by the proof of Theorem A. I claim that $\Psi_{s}=\Psi_{t}$. Proposition 3.7 reduces the problem to comparing the operations on a point and $\mathbb{C} P^{\infty}$. They coincide on a point since

$$
\Psi_{s}(p t)=\psi=\Psi_{t}(p t)
$$

They coincide on $\mathbb{C} P^{\infty}$ since

$$
\operatorname{Spf} \Psi_{s}\left(\mathbb{C} P^{\infty}\right)=\pi=\operatorname{Spf} \Psi_{t}\left(\mathbb{C} P^{\infty}\right) .
$$

5.2. Proof of Corollary B, Given Theorem $\mathrm{A}$ and the remarks preceding the statement of Corollary $[\mathrm{B}$, all that remains is to observe that if $N=|A|$, then the dual isogeny $\pi^{\vee}$ gives a factorization

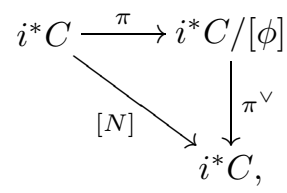

and that $[N]$ induces an isomorphism of formal groups over $R\left[\frac{1}{N}\right]$.

\section{EXAMPLES}

In this section we construct isogeny data, and so operations, for the theories based on the Igusa quartic, $E_{2}$, and the Tate curve. In the first two cases, the method is to observe that the elliptic curve $C \rightarrow S_{E}$ is universal for some property, and then to find an instance of that property on the quotient elliptic curve $D \rightarrow S_{F}$, which is classified by a map

$$
S_{F} \stackrel{\psi}{\longrightarrow} S_{E}
$$

In all three cases, there is a coordinate on the elliptic curve $C$ in terms of which the effect of $\Psi\left(\mathbb{C} P^{\infty}\right)$ is given by a norm. The appearance of this norm was foreshadowed by formulas for the $H_{\infty}$ structure on $M P$, in honor of which we call the operations $\Psi$ power operations.

6.1. Igusa. Let $(E, I)$ be the elliptic cohomology based on the Igusa quartic (section 2.1), let $N$ be an odd integer, and let

$$
S_{F}=\operatorname{Spec} F \stackrel{i}{\rightarrow} S_{E}
$$

be the scheme of Drinfel'd $(\mathbb{Z} / N)^{2}$-structures on $I$, with universal example

$$
(\mathbb{Z} / N)^{2} \stackrel{\phi}{\rightarrow} i^{*} I .
$$

For each subgroup

$$
A \subset(\mathbb{Z} / N)^{2}
$$

we obtain by restriction a level- $A$ structure $\phi_{A}$ which fits into a Drinfel'd isogeny

$$
A \stackrel{\phi_{A}}{\longrightarrow} i^{*} I \stackrel{\pi_{A}}{\longrightarrow} i^{*} I / A \stackrel{\text { def }}{=} i^{*} I /\left[\phi_{A}\right] .
$$

Write $\tilde{N}_{A} x$ for the function $\tilde{N}_{\phi_{A}} i^{*} x$ on $i^{*} I / A$. The first point is

Lemma 6.1. $\tilde{N}_{A} x$ is a coordinate on $i^{*} I / A$. 
Proof. Proposition 4.5 applies. The function $x$ is constructed to have divisor

$$
[2]^{-1}(e)-[2]^{-1}(P),
$$

where $P$ is a point of strict order 2 ; and this feature of $x$ is preserved under base change. If the order of $A$ is odd, then $x$ never has a pole at $\phi(a)$ for $a \in A$, and it vanishes at $\phi(a)$ if and only if $\phi(a)=e$.

Let $\psi^{A}$ and $g_{A}$ be the maps

$$
\begin{gathered}
S_{F} \stackrel{\psi^{A}}{\longrightarrow} S_{E} \\
i^{*} I / A \stackrel{g_{A}}{\longrightarrow} \psi^{A *} I^{\prime}
\end{gathered}
$$

associated to the triple $\left(i^{*} I / A, e^{*} d \tilde{N}_{A} x, \pi_{A}(P)\right)$ by the universal property of Igusa's quartic (see section 2). By construction we have

Theorem 6.2 (Theorem C). The pair $\left(\psi^{A}, \hat{g}_{A}\right)$ is a factorization of $\left(i, \pi_{A}\right)$.

Theorems $\$$ and 6.2 together construct an operation $\Psi^{A}$ for the elliptic cohomology given by the Igusa quartic. In fact, one can be quite explicit about the effect of $\Psi^{A}\left(\mathbb{C} P^{\infty}\right)$ in this case.

\section{Proposition 6.3.}

$$
\Psi^{A}\left(\mathbb{C} P^{\infty}\right)(x)=\widehat{N_{A} i(x)} .
$$

Proof. By construction we have

$$
\hat{\pi}_{A}^{*} \hat{g}_{A}^{*} \psi^{A *} x=\Psi^{A}\left(\mathbb{C} P^{\infty}\right)(x)
$$

and

$$
\hat{\pi}_{A}^{*} \tilde{N}_{A} x=\widehat{N_{A} i(x)} .
$$

We must show that $g_{A}^{*} \psi^{A *} x=\tilde{N}_{A} x$. Now $g_{A}^{*} \psi^{*} x$ is the function on $i^{*} I / A$ satisfying the equations

$$
\begin{aligned}
\operatorname{div} g_{A}^{*} \psi^{*} x & =[2]^{-1}(e)-[2]^{-1}\left(\pi_{A}(P)\right), \\
e^{*} d g_{A}^{*} \psi^{*} x & =e^{*} d \tilde{N}_{A} x .
\end{aligned}
$$

It is easy to check that $\tilde{N}_{A} x$ also satisfies these two equations.

Corollary 6.4. For $N$ odd there is an additive natural transformation

$$
T_{N}=\sum_{\substack{A \subset(\mathbb{Z} / N)^{2} \\|A|=N}} \Psi^{A}
$$

from $E^{0}(-)$ to $E^{0}(-)$.

Proof. The sum $\sum_{\substack{A \subset(\mathbb{Z} / N)^{2} \\|A|=N}} \Psi^{A}$ clearly gives an additive operation

$$
E^{0}(-) \stackrel{T_{N}}{\longrightarrow} F^{0}(-)
$$

or equivalently (since $E$ is $\mathcal{R} \mathcal{W}$ ) an element of $\operatorname{Hom}_{E}\left[E_{0}\left(\Omega^{\infty} E\right), F\right]$. What remains is to show that this element is in the subset

$$
\operatorname{Hom}_{E}\left[E_{0}\left(\Omega^{\infty} E\right), E\right] \subset \operatorname{Hom}_{E}\left[E_{0}\left(\Omega^{\infty} E\right), F\right] .
$$


Indeed it suffices to show that it lies in the subset

$$
\operatorname{Hom}_{E}\left[E_{0}\left(\Omega^{\infty} E\right), E\left[\frac{1}{N}\right]\right] \subset \operatorname{Hom}_{E}\left[E_{0}\left(\Omega^{\infty} E\right), F\left[\frac{1}{N}\right]\right] .
$$

Now Baker's argument Bak90] applies; the point is that $T_{N}(p t)$ is just $N$ times the classical Hecke operator.

6.2. Power operations. Suppose that $C_{0}$ is a formal group of height $n$ over a finite field $k$ of characteristic $p>0$, and let $E\left(C_{0}\right)$ be the resulting cohomology theory as in section 2. Then $C=G_{E\left(C_{0}\right)}$ is the Lubin-Tate deformation of $C_{0}$. Lubin and Tate LT65 show that over the ring of integers of the algebraic closure of the fraction field of $E\left(C_{0}\right)$ we have

$$
C_{\text {tors }} \cong\left(\left(\mathbb{Z} / p^{\infty}\right)^{n}\right) \text {. }
$$

Let $A$ be a finite subgroup of $\left(\mathbb{Z} / p^{\infty}\right)^{n}$ of order $q$, and let $i: E\left(C_{0}\right) \rightarrow E\left(C_{0}, A\right)$ be a universal complete local $E\left(C_{0}\right)$-algebra equipped with a level- $A$ structure

$$
A \stackrel{\phi}{\longrightarrow} i^{*} C \stackrel{\pi}{\longrightarrow} i^{*} C / A
$$

as in Proposition 4.3. Proposition 4.3 implies that $i$ is flat; let

$$
E\left(C_{0}, A\right)^{*}(-)=E\left(C_{0}, A\right) \underset{i}{\otimes} E\left(C_{0}\right)^{*}(-)
$$

be the resulting cohomology theory.

Since $C_{0}(k)=0$, the homomorphism $\phi$ induces the zero homomorphism over $k$. It follows that there is an isomorphism

$$
\left(i^{*} C / A\right)_{0} \cong C_{0}^{(q)}
$$

of formal groups over $k$, which identifies the isogeny $\pi_{0}$ with the $q$-power Frobenius.

It follows that $i^{*} C / A$ is a deformation of $C_{0}^{(q)}$. Letting $H$ over $E\left(C_{0}^{(q)}\right)$ be the universal deformation of the formal group $C_{0}^{(q)}$, it follows that there is a map of local rings

$$
E\left(C_{0}^{(q)}\right)(p t) \stackrel{\psi}{\longrightarrow} E\left(C_{0}, A\right)(p t)
$$

and an isomorphism of formal groups

$$
i^{*} C / A \stackrel{g}{\rightarrow} \psi^{*} H
$$

The data $(i, g \pi, \psi)$ produces, using Theorem $\mathrm{A}$, a ring operation

$$
E\left(C_{0}^{(q)}\right)^{0}(-) \stackrel{\Psi}{\longrightarrow} E\left(C_{0}, A\right)^{0}(-) .
$$

Moreover, if $k=\mathbb{F}_{p}$ so that $C_{0} \cong C_{0}^{(q)}$, then we have

$$
E\left(C_{0}\right)(-) \cong E\left(C_{0}^{(q)}\right)(-),
$$

and so an operation

$$
E\left(C_{0}\right)(-) \stackrel{\Psi}{\longrightarrow} E\left(C_{0}, A\right)^{0}(-)
$$

It is in this case that the relationship with the $H_{\infty}$ structure on $M P$ is easiest to explain. Let $t$ be any coordinate on $C$ such that $t(\phi(a)) \neq \infty$ for $a \in A$. $C_{0}(k)$ is the one-element group, so the condition of Proposition 4.5 is automatically satisfied. The resulting coordinate $\tilde{N}_{\phi} t$ on $i^{*} C / A$ yields an operation

$$
M P^{0}(-) \stackrel{Q}{\longrightarrow} E\left(C_{0}, A\right)^{0}(-)
$$

by Proposition 5.1 
Another construction of this operations goes as follows [And95]. The $H_{\infty}$ structure on $M P$ gives an operation

$$
M P^{0}(-) \stackrel{P}{\longrightarrow} M P^{0}\left(B A^{*} \times-\right)
$$

(where $A^{*}=\operatorname{Hom}\left[A, \mathbb{C}^{\times}\right]$), and the character theory of [HKR92] gives an operation

$$
E^{0}\left(B A^{*} \times-\right) \stackrel{H K R}{\longrightarrow} E\left(C_{0}, A\right)^{0}(-) .
$$

The composite

$$
M P^{0}(-) \stackrel{P}{\longrightarrow} M P^{0}\left(B A^{*} \times-\right) \stackrel{t}{\rightarrow} E^{0}\left(B A^{*} \times-\right) \stackrel{H K R}{\longrightarrow} E\left(C_{0}, A\right)^{0}(-)
$$

is $Q$.

In And95 we showed that one can choose the coordinate $t$ so that the diagram

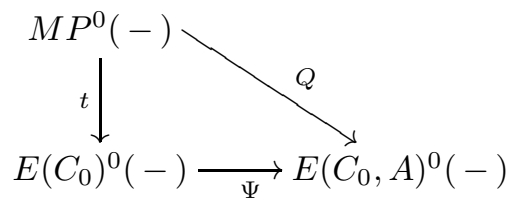

commutes, and so

$$
\Psi\left(\mathbb{C} P^{\infty}\right)(t)=N_{A} i(t),
$$

as in the case of the Igusa quartic. It is in honor of this relationship with the $H_{\infty}$ structure on $M P$ that we call the operations based on isogenies power operations.

6.3. The Tate curve. There are two basic families of isogenies out of the Tate curve. If $a$ is a positive integer, then there is an isogeny

$$
\operatorname{Tate}(q) \stackrel{[a]}{\longrightarrow} \operatorname{Tate}\left(q^{a}\right)
$$

with kernel $\mu[a]$, modeled on

$$
\begin{gathered}
F^{\times} / q^{\mathbb{Z}} \stackrel{[a]}{\longrightarrow} F^{\times} / q^{a \mathbb{Z}} \\
z \mapsto z^{a} .
\end{gathered}
$$

Over $\mathbb{Z}\left(\left(q^{1 / b}\right)\right)$ there is an isogeny

$$
\operatorname{Tate}(q) \stackrel{\pi_{b}}{\longrightarrow} \operatorname{Tate}\left(q^{1 / b}\right)
$$

with kernel $\mathbb{Z}\left[\frac{1}{b}\right] / \mathbb{Z}$, modeled on the projection

$$
\begin{aligned}
K^{\times} / q^{\mathbb{Z}} \stackrel{\pi_{b}}{\longrightarrow} K^{\times} / q^{\frac{1}{b} \mathbb{Z}} \\
z \mapsto z \bmod q^{\frac{1}{b}} .
\end{aligned}
$$

More generally, let $D(a, b)$ be the $A=\mathbb{Z}((q))$-algebra

$$
D(a, b)=A[r] /\left(r^{b}=q^{a}\right) .
$$

There are two ring homomorphisms

$$
\mathbb{Z}((q)) \underset{\psi}{\stackrel{i}{\rightrightarrows}} D(a, b),
$$

namely, $i(q)=q$ and $\psi(q)=r$, and an isogeny

$$
g_{a, b}: i^{*} \text { Tate }=\operatorname{Tate}(q) \stackrel{[a]}{\longrightarrow} \operatorname{Tate}\left(q^{a}\right) \stackrel{\pi_{b}}{\longrightarrow} \operatorname{Tate}(r)=\psi^{*} \text { Tate. }
$$


The kernel of $g_{a, b}$ is the $D(a, b)$-scheme

$$
T[a, b] \stackrel{\text { def }}{=}\left\{z \in T(q)[a b] \mid z^{a}=r^{i} \text { for some } i\right\} .
$$

It is a disjoint union of components

$$
T_{i}[a, b]=T[a, b] \cap T_{i a}[a b]
$$

for $i=0, \ldots, b-1$, and fits into a short exact sequence of the form

$$
\mu[a] \hookrightarrow T[a, b] \rightarrow \mathbb{Z}\left[\frac{1}{b}\right] / \mathbb{Z} .
$$

The following is easy to check using formulae which are available, for example, from [Sil94.

Lemma 6.5. The diagram

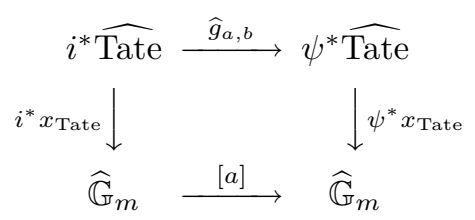

commutes.

Applying Theorem $\underline{\mathrm{A}}$, we have

Corollary 6.7. For $a, b \geq 1$ there is a ring operation

$$
D(a, b) \underset{i}{\hat{\otimes}} K_{\text {Tate }}(-) \stackrel{\Psi^{a, b}}{\longrightarrow} D(a, b) \underset{\psi}{\hat{\otimes}} K_{\text {Tate }}(-)
$$

such that, considering $x_{\text {Tate }}$ as an element of $K_{\text {Tate }}\left(\mathbb{C} P^{\infty}\right)$,

$$
\Psi^{a, b}\left(\mathbb{C} P^{\infty}\right)\left(\psi^{*} x_{\text {Tate }}\right)=[a]^{*} i^{*} x_{\text {Tate }} .
$$

In terms of the function $z=1-x_{\text {Tate }}$, we have

$$
\Psi^{a, b}\left(\mathbb{C} P^{\infty}\right)\left(\psi^{*} z\right)=\left(i^{*} z\right)^{a} .
$$

Although the coordinate $\theta(2.8)$ is not a function on the Tate curve, so Proposition 4.5 is not directly relevant, it is still true that the effect of $\Psi^{a, b}$ on $\theta$ is given by a suitable norm. We treat the cases $\Psi^{1, n}$ and $\Psi^{n, 1}$.

By equation (6.9), we have

$$
\Psi^{1, n}\left(\mathbb{C} P^{\infty}\right)(\theta(z, q))=\theta(z, r),
$$

while we check directly that

$$
N_{g_{1, n}} \theta(z, q)=\prod_{j=0}^{n-1} \theta\left(z r^{j}, q\right)=\theta(z, r) .
$$

Similarly,

$$
\Psi^{n, 1}\left(\mathbb{C} P^{\infty}\right)(\theta(z, q))=\theta\left(z^{n}, q^{n}\right)=\prod_{j=0}^{n-1} \theta\left(z \zeta^{j}, q\right)=N_{g_{n, 1}} \theta(z, q),
$$

where $\zeta$ is a primitive $n^{\text {th }}$ root of 1 . 
Remark 6.12. In fact, $\theta$ is a section of the line bundle associated to the divisor $(e)$ over Tate; see Remark 10.11 and the discussion of the basic representation of $L \mathbb{T}$ in section 11 The formulae here describe the behavior of this line bundle under the norm maps defined by various isogenies.

Remark 6.13. Let $\zeta$ denote a primitive $b^{\text {th }}$ root of unity. If $R$ is a $\mathbb{Z}[\zeta]((q))$-algebra and $r \in R$ satisfies $r^{b}=q^{a}$, then the $R$-valued points of $\operatorname{Spec} D(a, b)$ are just

$$
(\operatorname{Spec} D(a, b))(R)=\left\{r \zeta^{j} \mid 0 \leq j<b-1\right\} ;
$$

the resulting isogenies are of the form

$$
\operatorname{Tate}(q) \rightarrow \operatorname{Tate}\left(q^{a}\right) \rightarrow \operatorname{Tate}\left(r \zeta^{j}\right) .
$$

We use the notation $\Psi^{a, b, \zeta^{j}}$ for the associated operation. For consistency with the discussion in Part III it will be convenient to study $D(1, b)$ under the ring homomorphism

$$
\begin{gathered}
\mathbb{Z}((q)) \stackrel{s_{b}}{\longrightarrow} \mathbb{Z}((q)) \\
q \mapsto q^{b} .
\end{gathered}
$$

This map extends over $i: \mathbb{Z}((q)) \rightarrow D(1, b)$ by

$$
s_{b}(r)=q \text {. }
$$

Indeed for $\zeta^{b}=1$ there is an extension of

$$
s_{b}: \mathbb{Z}[\zeta]((q)) \rightarrow \mathbb{Z}[\zeta]((q))
$$

given by

$$
s_{b, \zeta}(r)=\zeta q .
$$

Under this specialization, the isogeny $g_{1, b}$ becomes

$$
g_{1, b, \zeta}: \operatorname{Tate}\left(q^{b}\right) \rightarrow \operatorname{Tate}(\zeta q)
$$

\section{Part II. Elliptic cohomology and loop groups}

\section{Notation}

We will make use of the following notation and conventions.

$$
\begin{aligned}
\mathbb{G}_{m} & :=\text { the multiplicative group } \operatorname{Spec} \mathbb{Z}\left[t, t^{-1}\right], \\
\mathbb{T} & :=\text { the circle group, written multiplicatively, } \\
T & :=\text { a compact torus, } \\
\hat{T} & :=\operatorname{Hom}[T, \mathbb{T}], \\
\check{T} & :=\operatorname{Hom}[\mathbb{T}, T] .
\end{aligned}
$$

We honor the tradition of identifying $\check{T}$ and $\hat{T}$ with lattices in the Lie algebra of $T$ and its $\mathbb{R}$-linear dual, by writing the group structure additively. More generally, if $L$ is a lattice (finitely generated free abelian group) and

$$
L^{\vee}:=\operatorname{Hom}[L, \mathbb{Z}]
$$

is its dual, then we frequently make use of the canonical isomorphisms

$$
\begin{aligned}
L & \cong \operatorname{Hom}\left[\mathbb{G}_{m}, L \otimes \mathbb{G}_{m}\right], \\
L^{\vee} & \cong \operatorname{Hom}\left[L \otimes \mathbb{G}_{m}, \mathbb{G}_{m}\right] .
\end{aligned}
$$


The point is that the right-hand side in each case is a constant group scheme.

If $A$ is an abelian group written additively, and $M$ is an abelian group written multiplicatively, we shall observe the tradition of writing $m^{a}$ for the element $a \otimes m$ of $A \otimes M$.

Elaborating on these traditions, if $n \in L^{\vee}$ and $z \in L \otimes \mathbb{G}_{m}$, we write $z^{n}$ for the resulting element of $\mathbb{G}_{m}$, etc.

\section{EquiVARIANT ELLIPTIC COHOMOLOGY}

If $G$ is a compact Lie group, denote by

$$
\mathbf{K}_{G}(-) \stackrel{\text { def }}{=} \operatorname{Spec} K_{G}(-)
$$

the spectrum of $G$-equivariant $K$-theory; it is a functor to $\mathbf{K}_{G}=\mathbf{K}_{G}(p t)=$ Spec $R G$-schemes.

The isomorphism

$$
\operatorname{Spf} K(B \mathbb{T}) \cong \widehat{\mathbb{G}}_{m}
$$

is the completion of the isomorphism

$$
\mathbf{K}_{\mathbb{T}}=\operatorname{Spec} R \mathbb{T}=\operatorname{Spec} \mathbb{Z}[\hat{\mathbb{T}}] \cong \mathbb{G}_{m} .
$$

More generally, a celebrated theorem of Atiyah-Segal says that if $T$ is a compact torus, then $K(B T)$ is a completion of

$$
\mathbf{K}_{T} \cong \check{T} \otimes \mathbb{G}_{m},
$$

and if $G$ is a connected compact Lie group, $T$ a maximal torus, and $W$ its Weyl group, then $\operatorname{Spf} K(B G)$ is a completion of

$$
\mathbf{K}_{G}=\operatorname{Spec} R G \cong\left(\check{T} \otimes \mathbb{G}_{m}\right) / W .
$$

If $(E, C, \kappa)$ is an elliptic spectrum, then

$$
\operatorname{Spf} E(B \mathbb{T}) \cong \hat{C}
$$

and it has always seemed desirable to construct an equivariant theory for which

$$
\mathbf{E}_{\mathbb{T}} \cong C,
$$

whose completion is (8.3). The difficulty is that the elliptic curve $C$ has no nonconstant holomorphic functions. Grojnowski and Ginzburg, Kapranov and Vasserot address this problem by ignoring it: $\mathbf{E}_{\mathbb{T}}$ should be a functor from $\mathbb{T}$-spaces to sheaves over $C$. Generalizing (8.2), $\mathbf{E}_{G}$ should be a functor from $G$-spaces to $W$-equivariant sheaves over

$$
\check{T} \otimes C .
$$

Grojnowski has constructed such a theory, based on the elliptic curve $\mathbb{C} /(\mathbb{Z}+\tau \mathbb{Z})$ over the upper half-plane.

Here we shall study the equivariant extension of $K_{\text {Tate }}$. It appears likely that Grojnowski's method carries over to this case, although we shall not address that problem here: we will be concerned only with

$$
\left(\mathbf{K}_{\text {Tate }}\right)_{G}=\left(\mathbf{K}_{\text {Tate }}\right)_{G}(p t)=(\check{T} \otimes \text { Tate }),
$$

with its $W$-action. From now on we write $\mathbf{E}=\mathbf{K}_{\text {Tate }}$.

It turns out that characters of representations of the loop group $L G$ are precisely the invariant sections of a canonical $W$-equivariant line bundle over $\mathbf{E}_{G}$. We 
reiterate that this is really a simple consequence of results of Kac and Looijenga, communicated to the author by Grojnowski; see the remarks at the end of the introduction. Starting from a recollection about representations from [PS86], we assemble results from [Loo76] and [Kac85] to arrive at Theorem D This much detail provides a foundation for the discussion in Part III

\section{REPRESENTATIONS OF LOOP GROUPS}

We denote by

$$
L G \stackrel{\text { def }}{=} \mathrm{C}^{\infty}\left[S^{1}, G\right]
$$

the group of smooth unbased loops in a compact connected Lie group $G$. Once we settle on an appropriate notion of representation for loop groups, the representation theory bears strong resemblance to the representation theory of compact Lie groups. We indicate some highlights, which we learned from [PS86].

Before describing the representations, note that:

(i) The group $G$ includes as the constant loops in $L G$.

(ii) The circle group $\mathbb{T}$ acts on $L G$ by rotating loops.

We write $\mathbb{T}_{\text {rot }}$ for the circle group acting in this way. Fix once and for all a generator $q$ of $\hat{\mathbb{T}}_{\text {rot }}$, so that

$$
R\left(\mathbb{T}_{\text {rot }}\right) \cong \mathbb{Z}\left[q, q^{-1}\right] .
$$

For $a \in \mathbb{Z}$ we shall denote by $L G \rtimes a$ the semi-direct product formed from the action

$$
\mathbb{T} \stackrel{a}{\rightarrow} \mathbb{T} \stackrel{\text { rot }}{\longrightarrow} \operatorname{Aut}(L G)
$$

The representations of $L G$ studied in [PS86] are projective representations of a definite level: let

$$
\mathbb{T} \rightarrow E \rightarrow L G
$$

be a central extension of $L G$ by the circle group $\mathbb{T}$; we write $\mathbb{T}_{\text {cent }}$ for the central circle.

Definition 9.1. A $G$-split central extension $E$ is a central extension of $L G$ by $\mathbb{T}$, together with

(i) a splitting $\left.E\right|_{G} \cong \mathbb{T}_{\text {cent }} \times G$ of the central extension over the constant loops; and

(ii) an action of $\mathbb{T}_{\text {rot }}$ on $E$ lifting the action on $L G$.

If $E$ is $G$-split, then we may form the semi-direct product $E \rtimes a$. Given a continuous action of $E \rtimes a$ on a topological vector space $V$, the subspace of "vectors of energy $k$ " is the vector space

$$
V(k) \stackrel{\text { def }}{=}\left\{v \in V \mid \mathbb{T}_{\text {rot }} \text { acts by the character } q^{k}\right\} .
$$

Definition 9.2. Let $E$ be a $G$-split central extension of $L G$. A representation of $L G$ of level $E$ and type $a$ is a unitary representation of $E \rtimes a$ on a separable Hilbert space $V$, such that

(i) for $k$ sufficiently small, $V(k)=0$;

(ii) each $V(k)$ is finite dimensional;

(iii) the center $\mathbb{T}_{\text {cent }}$ of the extension acts by the standard character of $\mathbb{T}_{\text {cent }}$. 
Remark 9.3. The definition of representation really gives a particular class of representations of $E$. However, if $G$ is simple and simply connected, a torus, or the unitary group $U_{r}$, there is a natural choice of central extension $E$, denoted $\tilde{L} G$ in [PS86]; see also sections [10 and 11] of the present paper. The level of generality here is intended to indicate more clearly the connection with theta functions in section 9.1 .

Remark 9.4. The restriction (iii) is not standard but also not serious. What in [PS86] might be called a representation of $E$ of level $h$ would here be called a representation of level $h_{*} E$.

Remark 9.5. The condition (i) of Definition 9.2 says that $V$ has "bounded negative energy". In [PS86] one restricts to $V$ such that $V(k)=0$ for $k<0$. The restriction is not serious since if $V$ has bounded negative energy, then there is a $k$ such that $q^{k} \otimes V$ has positive energy.

Definition 9.6. An essential equivalence [PS86, Chapter 9.3] from $V$ to $W$ is a continuous equivariant inclusion $V \rightarrow W$ with dense image.

With these restrictions, the representation theory of $L G$ resembles closely the representation theory of a compact Lie group.

(i) Every representation is essentially equivalent to a discrete direct sum of irreducible representations. We shall say that two representations $V$ and $W$ are equivalent if there are a discrete direct sum of irreducible representations $D=\bigoplus_{\alpha \in I} V_{\alpha}$ and essential equivalences

$$
V \hookleftarrow D \hookrightarrow W .
$$

(ii) The equivalence class of a representation is determined by its restriction to the maximal torus $\mathbb{T}_{\text {cent }} \times T \times \mathbb{T}_{\text {rot }}$. A representation $V$ contains, as a dense subspace, the sum

$$
\bigoplus_{(v, n) \in \hat{T} \times \hat{\mathbb{T}}_{\text {rot }}} V_{(v, n)}
$$

of subspaces on which this maximal torus acts by the indicated character. This direct sum is recorded in terms of its character

$$
\chi_{V}(t, z, q)=t \sum_{(v, n)} \operatorname{dim} V_{(v, n)} q^{n} z^{v} .
$$

A priori this is merely a suggestive way to record the map of sets

$$
\begin{aligned}
\hat{T} \times \hat{\mathbb{T}}_{\text {rot }} & \rightarrow \mathbb{Z} \\
(v, n) & \mapsto \operatorname{dim} V_{(v, n)} .
\end{aligned}
$$

The requirements that $V$ have bounded negative energy and that $V(k)$ is finitedimensional for each $k$ guarantee that $\chi_{V}$ may be viewed as an element of the ring

$$
t(\mathbb{Z}[\hat{T}]) \llbracket q \rrbracket
$$

(the notation $\mathbb{Z}[\hat{T}]$ refers to the group ring on the character group etc.; the $t$ records the fixed character by which $\mathbb{T}_{\text {cent }}$ acts). 
Remark 9.7. The restriction (iii) of Definition 9.2 is also not standard. However, if representations are defined without it, then the irreducible representations still satisfy (iii) of 9.2, and every representation is essentially equivalent to a sum of irreducible representations. The reason for the restriction is to make it easy to define the tensor product of representations; see the discussion of Adams operations in section 12.

Definition 9.8. Denote by $\operatorname{Rep}_{(a, E)}(L G)$ the Grothendieck group of equivalence classes of representations of $L G$ of level $E$ and type $a$.

9.1. Relationship to theta functions. The group $\mathbb{T}_{\text {cent }} \times T \times \mathbb{T}_{\text {rot }}$ is a maximal torus of $E \rtimes a$ for any $a$; we write $\mathbb{T}_{\text {cent }} \times T \times a$ if we wish to distinguish the group in which it sits. Let

$$
W_{\text {aff }}(a) \stackrel{\text { def }}{=} N\left(\mathbb{T}_{\text {cent }} \times T \times a\right) / \mathbb{T}_{\text {cent }} \times T \times a
$$

be its Weyl group; it is called the affine Weyl group. It acts on the maximal torus, and the character $\chi_{V}$ is invariant. The link between the representation theory of $L G$ and the space $\mathbf{E}_{G}$ comes from this invariance.

Before studying $W_{\text {aff }}(a)$ and its action on the maximal torus, observe that we can identify $\check{T}=\operatorname{Hom}[\mathbb{T}, T]$ with a subgroup of $L G$.

Lemma 9.9. Let $W$ be the Weyl group of $T$ in $G$. Then the Weyl group $W_{\text {aff }}(a)$ is isomorphic to $W \ltimes \check{T}$. The element $n \in \check{T}$ acts on $T \times a$ by the formula

$$
n \cdot(z, q)=\left(q^{a n} z, q\right) \text {. }
$$

Proof. This is standard. For example, Proposition (5.1.2) in PS86] shows that $W_{\text {aff }}$ is the Weyl group of $T \times \mathbb{T}_{\text {rot }}$ in $L G \rtimes \mathbb{T}_{\text {rot }}$. It is easy to see that passing to $L G \rtimes a$ has no effect on the argument. As $E$ is a central extension, the action lifts to $E$, and the Weyl group can be no larger. The formula for the action of $\check{T}$ on $T \times a$ is

$$
\begin{aligned}
n(z, q)(-n) & =(n, 1)(z, q)(n,-1) \\
& =\left(\theta \mapsto\left(q^{a} \theta\right)^{n} z\left(\theta^{-n}\right), q\right) \\
& =\left(\theta \mapsto q^{a n} z, q\right) \\
& =\left(q^{a n} z, q\right) .
\end{aligned}
$$

Because of Lemma 9.9 we drop the $a$ and revert to the usual notation $W_{\text {aff }}$ for the affine Weyl group.

Definition 9.10. If $A$ and $B$ are abelian groups then a map $\phi: A \rightarrow B$ of degree 2 is a map of sets such that

$$
\phi(x+y+z)-\phi(x+y)-\phi(x+z)-\phi(y+z)+\phi(x)+\phi(y)+\phi(z)-\phi(0)=0 .
$$

It is pointed if in addition $\phi(0)=0$.

Proposition 9.11. There are a $W$-invariant pointed map of degree 2

$$
\phi_{(a, E)}: \check{T} \rightarrow \mathbb{Z}
$$

and a $W$-invariant symmetric bilinear map

$$
I_{(a, E)}: \check{T} \times \check{T} \rightarrow \mathbb{Z}
$$


related by the formula

$$
\phi(u+v)=\phi(u)+\phi(v)+a I(u, v),
$$

such that the action of $n \in \check{T}$ on $\mathbb{T}_{\text {cent }} \times T \times a$ is given by the formula

$$
n \cdot(t, z, q)=\left(q^{\phi(n)} z^{I(n)} t, q^{a n} z, q\right) .
$$

In the formula, $I(n)$ refers to the element of $\hat{T}=\operatorname{Hom}[\check{T}, \mathbb{Z}]$ obtained from the adjoint of the bilinear map $I$.

Proof. Certainly there is some pair of pointed functions

$$
\begin{aligned}
& \phi: \check{T} \rightarrow \mathbb{Z} \\
& \tilde{I}: \check{T} \rightarrow \hat{T}
\end{aligned}
$$

such that

$$
n \cdot(t, z, q)=\left(q^{\phi(n)} z^{\tilde{I}(n)} t, q^{a n} z, q\right) .
$$

The associativity and commutativity of the action first give that $\tilde{I}$ is a homomorphism, and so the adjoint of a bilinear map $I$, and second that $\phi$ and $I$ are related by (9.12). The $W$-invariance can be seen by examining the equation

$$
(w n) \cdot(t, w \cdot z, q)=w \cdot(n \cdot(t, z, q))
$$

Corollary 9.14. If $V$ is a representation of level $E$ and type $a$, then

$$
\chi_{V}\left(t, q^{a n} z, q\right)=q^{-\phi(n)} z^{-I(n)} \chi_{V}(t, z, q) .
$$

Proof. Since $\chi_{V}$ is a character we have

$$
\chi_{V}(n \cdot(t, z, q))=\chi_{V}(t, z, q) .
$$

The left-hand side is

$$
\chi_{V}\left(q^{\phi(n)} z^{I(n)} t, q^{a n} z, q\right) .
$$

Since $\mathbb{T}_{\text {cent }}$ acts by its standard character, this is in turn is equal to

$$
q^{\phi(n)} z^{I(n)} \chi_{V}\left(t, q^{a n} z, q\right) .
$$

Equation (9.15) is typical of the transformation formulae of theta functions. Namely, recall the notations

$$
\begin{aligned}
\mathbf{K}_{T} & =\check{T} \otimes \mathbb{G}_{m}, \\
\mathbf{E}_{T} & =\check{T} \otimes \text { Tate. }
\end{aligned}
$$

Let $F$ be a $p$-adic field or $\mathbb{C}$, and suppose that $q \in F^{\times}$is an element with $|q|<1$. The exact sequence

$$
1 \rightarrow q^{a \mathbb{Z}} \rightarrow \mathbb{G}_{m}(F) \rightarrow \operatorname{Tate}\left(q^{a}\right)(F) \rightarrow 1
$$

of Theorem 2.7 results in an exact sequence

$$
1 \rightarrow q^{a \breve{T}} \rightarrow \mathbf{K}_{T}(F) \rightarrow \mathbf{E}_{T}\left(q^{a}\right)(F) \rightarrow 1,
$$

where the group

$$
q^{a \check{T}}=\{a n \otimes q \mid n \in \check{T}\}
$$


is isomorphic to the subgroup

$$
\check{T} \subset W_{\text {aff }} .
$$

Proposition 9.11 says that the action of $n \in \check{T}$ on $\mathbf{K}_{T}(F)$ is translated by $q^{a n}$.

Define

$$
\check{T} \times \mathbf{K}_{T}(F) \stackrel{e}{\rightarrow} \mathbb{G}_{m}(F)
$$

by the formula

$$
e(n, z)=q^{-\phi(n)} z^{-I(n)}
$$

Then the formula

$$
n \cdot(t, z)=\left(e(n, z) t, z q^{a n}\right)
$$

defines an action of $\check{T}$ on $F \times \mathbf{K}_{T}(F)$ covering the action on $\mathbf{K}_{T}(F)$, and gives rise to a line bundle

$$
\frac{F \times F^{\times}}{(t, z) \sim\left(e(n, z) t, z q^{a n}\right)}
$$

over $\mathbf{E}_{T}\left(q^{a}\right)$. A section of this line bundle is a function $f$ on $\mathbf{K}_{T}(F)$ which satisfies

$$
f\left(z q^{a n}\right)=e(n, z) f(z) .
$$

Definition 9.20. Let $\mathcal{L}(a, I, \phi)$ be a line bundle over $\mathbf{E}_{T}\left(q^{a}\right)$ given by (9.18). Let $\Gamma(a, I, \phi)$ be the group of power series

$$
f \in(\mathbb{Z}[\hat{T}])((q))
$$

which satisfy

$$
f\left(z q^{a n}\right)=q^{-\phi(n)} z^{-I(n)} f(z, q)
$$

for $n \in \check{T}$ (see the appendix). If $E$ is a central extension of a loop group $G$, then we write $\mathcal{L}(a, E)$ for $\mathcal{L}\left(a, I_{(a, E)}, \phi_{(a, E)}\right)$ and $\Gamma(a, E)$ for $\Gamma\left(a, I_{(a, E)}, \phi_{(a, E)}\right)$.

Suppose that $F$ is a non-archimedean field and $q$ is a unit of $F$ with $|q|<1$. Let $\Gamma(\mathcal{L}(a, E))(q)$ denote the global analytic sections of the resulting line bundle over $\check{T} \otimes \operatorname{Tate}(q)$. In the cases of interest, it turns out that

$$
\Gamma(\mathcal{L}(a, E))(q) \cong F \underset{\mathbb{Z}((q))}{\otimes} \Gamma(a, E) ;
$$

see Theorems 10.10 and 11.6

In any case, the map which assigns to a representation of $L G$ of level $E$ and type $a$ its character, may be viewed as a homomorphism of $\mathbb{Z}((q))$-modules

$$
\operatorname{Rep}_{(a, E)}(L G) \stackrel{\chi}{\rightarrow} \Gamma(a, E) .
$$

Of course, the characters are also invariant under the action of the Weyl group $W$ of $G$. This group acts on $\Gamma(a, E)$ via its action on $\hat{T}$, and we denote by $\Gamma(a, E)^{W}$ the subgroup of power series which are invariant under this action. We summarize the proceeding discussion as

Proposition 9.21. The character may be viewed as a homomorphism of $\mathbb{Z}((q))$ modules

$$
\operatorname{Rep}_{(a, E)}(L G) \stackrel{\chi}{\rightarrow} \Gamma(a, E)^{W}
$$


The Kac character formula shows that this map is often an isomorphism. We treat the case of simple and simply connected groups in section 10 and the case of a compact torus in section [1].

\section{Simple AND SIMPLY CONNECTED GROUPS}

10.1. The universal central extension. When $G$ is simple and simply connected, any central extension $E$ of $L G$ is $G$-split, and is completely determined by the bilinear form $I(1, E)$ of Proposition 9.11. In fact, there is a universal central extension $\tilde{L G}$ such that any other is canonically isomorphic to $h_{*} \tilde{L G}$ for some $h$, and the central extension $\tilde{L} G$ is canonically $G$-split. The bilinear form $I$ associated to $\tilde{L G}$ is the smallest $W$-invariant bilinear form $I$ such that $I(n, n) \in 2 \mathbb{Z}$ for $n \in \check{T}$ (see [Loo76], PS86, §4.4]), and the map $\phi$ is just

$$
\phi(n)=\frac{1}{2} I(n, n) .
$$

Definition 10.1. Suppose that $G$ is simple and simply connected. A representation of $L G$ of level $k$ is a representation of $L G$ of level $k_{*} \tilde{L G}$ and type 1 .

It is natural in this case to consider a representation of level $k$ as a representation of $\tilde{L G}$ on which $\mathbb{T}_{\text {cent }}$ acts by $\mathbb{T}_{\text {cent }} \stackrel{k}{\rightarrow} \mathbb{T}$, and to write the character as

$$
\chi(t, z, q)=t^{k} \sum_{v, n} \operatorname{dim} V_{(v, n)} z^{v} q^{n} .
$$

We use the abbreviations

$$
\begin{aligned}
\operatorname{Rep}_{k}(L G) & =\operatorname{Rep}_{\left(1, k_{*} \tilde{L G}\right)}(L G), \\
\mathcal{L}(G) & =\mathcal{L}(1, \tilde{L G}), \\
\Gamma(G)_{k} & =\Gamma\left(1, k_{*} \tilde{L G}\right) .
\end{aligned}
$$

For $k \in \mathbb{Z}$ the line bundle $\mathcal{L}\left(1, k_{*} \tilde{L} G\right)$ is just $\mathcal{L}(1, G)^{k}$, and $\Gamma(G)_{k}$ is the group of functions

$$
f \in(\mathbb{Z} \llbracket \hat{T} \rrbracket)((q))
$$

which satisfy

$$
f\left(z q^{n}, q\right)=q^{-k \phi(n)} z^{-k I(n)} f(z, q)
$$

for $n \in \check{T}$.

For $v \in \hat{T}$, let $g_{v, k}=g_{v, k, I}$ be the function

$$
g_{v, k}(z, q) \stackrel{\text { def }}{=} \sum_{n \in \check{T}} q^{k \phi(n)+\langle n, v\rangle} z^{k I(n)+v} .
$$

Then according to Lemma A.8 $g_{v, k}$ is an element of $\Gamma(G)_{k}$, and Proposition A.9 becomes

Proposition 10.2. If $S$ is a set of coset representatives for $\hat{T} / k \check{T}$, then the functions $g_{s, k}$ for $s \in S$ are a basis for $\Gamma(G)_{k}$ over $\mathbb{Z}((q))$. If $F$ is a non-archimedean field and $q \in F^{\times}$with $|q|<1$, then

$$
\Gamma\left(\mathcal{L}(G)^{k}\right)(q) \cong F \underset{\mathbb{Z}((q))}{\otimes} \Gamma(G)_{k}
$$


10.2. Sections invariant under the Weyl group. Recall that $\Gamma(G)_{k}^{W}$ is the submodule of $\Gamma(G)_{k}$ which is fixed under the action of the Weyl group $W$ of $T$ in $G$. We similarly define $\Gamma(G)_{k}^{-W}$ to be the submodule of $W$-anti-invariant elements sections, i.e., elements $s$ such that $w^{*} s=\operatorname{det}(w) s$ for $w \in W$. Proposition 10.2 parametrizes a basis for $\Gamma(G)_{k}$ by a fundamental domain for the action of $k \check{T}$ on $\hat{T}$ (via the inclusion induced by $I$ ). We obtain a basis for $\Gamma_{k}^{ \pm W}$ once we have a fundamental domain for the action of $W$ on $\hat{T} / k \check{T}$, or, equivalently, a fundamental domain for the action of $W_{\text {aff }}$ on $\hat{T}$. The "fundamental alcove" is a fundamental domain.

The results described from this point until the character formula were obtained in Loo76].

10.3. The fundamental alcove and the highest weight. Let $R \subset \hat{T}$ be the roots of $G$, let $R_{+}$be a set of positive roots, let $\alpha_{1}, \ldots, \alpha_{r}$ be a basis of for $R$ consisting of positive roots, and let $\alpha_{i}^{\vee} \in \check{T}$ be the coroot corresponding to $\alpha_{i}$. The subset $C h \subset \hat{T}$ given by

$$
C h:=\left\{v \mid\left\langle v, \alpha_{i}^{\vee}\right\rangle \geq 0,1 \leq i \leq r\right\} .
$$

is a fundamental domain for the action of $W$ on $\hat{T}$.

Let $\tilde{\alpha}$ be the highest root. The fundamental alcove of level $k$ is the simplex (in $C h)$

$$
P_{k}^{+}=\left\{v \in \hat{T} \mid\left\langle v, \alpha_{i}^{\vee}\right\rangle \geq 0 \text { and } I(\tilde{\alpha}, v)<k\right\} .
$$

Proposition 10.3. The set $P_{k}^{+}$is a fundamental domain for the action of $W \ltimes k \check{T}$ on $\hat{T}$.

For $\lambda \in P_{k}^{+}$define

$$
A_{\lambda, k}(z):=\sum_{w \in W} \operatorname{det}(w) g_{\lambda, k}(w \cdot z) .
$$

Theorem 10.4. The functions $A_{\lambda, k}$ form a $\mathbb{Z}((q))$-basis for $\Gamma(G)_{k}^{-W}$.

In order to describe the important basis for $\Gamma(G)_{k}^{W}$, we need a little more notation.

1. Let $\tilde{\alpha}^{\vee}$ be the coroot corresponding to $\tilde{\alpha}$.

2. Define non-negative integers $g_{i}$ by

$$
\tilde{\alpha}^{\vee}=g_{1} \alpha_{1}^{\vee}+\cdots+g_{r} \alpha_{r}^{\vee} .
$$

3. Set

$$
g=1+\sum g_{i}
$$

4. Let $\left\{\mu_{i}\right\}$ be the basis of $\hat{T}$ dual to $\left\{\alpha_{i}^{\vee}\right\}$.

5. Let $\rho=\sum \mu_{i}$.

6. Let

$$
\Phi(q)=\prod_{n \geq 1}\left(1-q^{n}\right)
$$

In terms of these, let

$$
A(z, q)=z^{\rho} \Phi(q)^{r} \cdot \prod_{\alpha \in R_{+}}\left(1-z^{-\alpha}\right) \cdot \prod_{n \geq 1} \prod_{\alpha \in R}\left(1-q^{n} z^{\alpha}\right) .
$$


Proposition 10.7. The function $A$ is an element of $\Gamma(G)_{g}^{-W}$. It freely generates $\Gamma(G)_{*}^{-W}$ as a module over $\Gamma(G)_{*}^{W}$.

10.4. The Kac character formula. The set $P_{k}^{+}$also parametrizes the irreducible representations of $L G$ of level $k$. For $\lambda \in P_{k}^{+}$, the Kac character formula records the character $\chi_{\lambda, k}$ of the representation corresponding to $\lambda$.

Kac Character Formula 10.8. The character of the representation of level $k$ corresponding to $\lambda \in P_{k}^{+}$is

$$
\chi_{\lambda, k}(t, z, q)=t^{k} A_{\lambda+\rho, k+g} / A .
$$

See, for example, chapter 13 of Kac85.

Corollary 10.9. If $G$ is a simple and simply connected group, then the character map induces an isomorphism

$$
\operatorname{Rep}_{k}(L G) \cong \Gamma(G)_{k}^{W}
$$

Proof. Theorem 10.8 together with Theorem 10.4 and Proposition 10.7 show that the characters $\chi_{\lambda, k}$ for $\lambda \in P_{k}^{+}$are a $\mathbb{Z}((q))$-basis for the space $\Gamma(G)_{k}^{W}$ of $W$-invariant elements of $\Gamma(G)_{k}$.

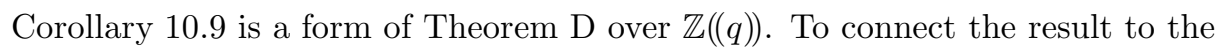
Tate curve, suppose that $F$ a is complete non-archimedean field, and $q$ is a unit of $F$ with $|q|<1$. Let $\mathcal{L}(G)(q)$ be the resulting $W$-equivariant line bundle over $\mathbf{E}_{T}(q)$, and let $\mathbf{E}_{G}\left(-; \mathcal{L}(G)^{k}\right)(q)$ denote Grojnowski's equivariant elliptic cohomology with coefficients in $\mathcal{L}(G)^{k}(q)$. Corollary 10.9 together with Proposition A.10 gives

Theorem 10.10. If $G$ is a simple and simply connected Lie group, then the character map establishes an isomorphism

$$
F \underset{\mathbb{Z}((q))}{\otimes} \operatorname{Rep}_{k}(L G) \cong \Gamma \mathbf{E}_{G}\left(p t, \mathcal{L}(G)^{k}\right)(q) .
$$

Remark 10.11. Because the formal group of the Tate curve is $\widehat{\mathbb{G}}_{m}$, its behavior in homotopy theory is essentially that of $K$-theory. It is encouraging that the line bundle $\mathcal{L}(G)$, or at least its divisor class, can be given over $\check{T} \otimes C$, where $C$ is any elliptic curve. We give a brief explanation of this fact, for which we are indebted to Mike Hopkins and the referee.

Each root $\alpha$ of $G$ determines a homomorphism

$$
\check{T} \otimes C \stackrel{\alpha \otimes C}{\longrightarrow} C
$$

whose kernel is a divisor $[\alpha]$ on $\check{T} \otimes C$. Let $\Delta$ be the $W$-invariant divisor

$$
\Delta=\sum_{\alpha \in R_{+}}[\alpha] .
$$

Looijenga [Loo76] observes that the divisor class of $\Delta$ is the same as that of the line bundle $\mathcal{L}(G)^{g}$, where $g$ is defined in (10.5). M. Hopkins and the referee have pointed out that the divisor class of $\mathcal{L}(G)$ is also well defined over any elliptic curve. Namely, let

$$
\hat{T} \stackrel{d}{\rightarrow} \operatorname{Pic}(\check{T} \otimes C)
$$


be the map

$$
d(\lambda)=(\lambda \otimes C)^{*}(e) .
$$

In other words, if $\mathcal{O}(-e)$ is the line bundle over $C$ associated to the divisor $(e)$, then $d(\lambda)$ is the divisor associated to the line bundle $(\lambda \otimes C)^{*} \mathcal{O}(-e)$.

Lemma 10.12. The map $d$ is a pointed map of degree 2 as in Definition 9.10. we have

$$
\begin{aligned}
d\left(\lambda_{1}+\lambda_{2}+\lambda_{3}\right)+ & d\left(\lambda_{1}\right)+d\left(\lambda_{2}\right)+d\left(\lambda_{3}\right) \\
& -d\left(\lambda_{1}+\lambda_{2}\right)-d\left(\lambda_{1}+\lambda_{3}\right)-d\left(\lambda_{2}+\lambda_{3}\right)-d(0)=0
\end{aligned}
$$

and

$$
d(0)=0 .
$$

Proof. The second equation is obvious. The first follows from the theorem of the Cube. If $\mathcal{L}$ is any line bundle over an elliptic curve $C$, let $\Theta(\mathcal{L})$ be the line bundle over $C^{3}$ whose fiber at $(x, y, z) \in E$ is

$$
\Theta(\mathcal{L})_{(x, y, z)} \stackrel{\text { def }}{=} \frac{\mathcal{L}_{x+y+z} \mathcal{L}_{x} \mathcal{L}_{y} \mathcal{L}_{z}}{\mathcal{L}_{x+y} \mathcal{L}_{x+z} \mathcal{L}_{y+z} \mathcal{L}_{0}} .
$$

The theorem of the cube implies that this line bundle is trivial. In particular, we may take $\mathcal{L}=\mathcal{O}(-e)$; then the left-hand side of (10.13) is just $\left(\lambda_{1}, \lambda_{2}, \lambda_{3}\right)^{*} \Theta(\mathcal{L})$.

Now if $G$ and $H$ are abelian groups, then the set of pointed maps of degree 2

$$
G \rightarrow H
$$

is isomorphic to

$$
\operatorname{Hom}\left[\operatorname{Sym}^{2}(G), H\right]
$$

where $\operatorname{Sym}^{2}(G)$ is the degree-two subgroup of the symmetric algebra on $G$; the universal map is

$$
\begin{aligned}
G \stackrel{u}{\longrightarrow} \operatorname{Sym}^{2}(G) \\
g \mapsto g \otimes g
\end{aligned}
$$

(see [Bre83, §8]). By Lemma10.12, the map

$$
\hat{T} \stackrel{d}{\rightarrow} \operatorname{Pic}(\check{T} \otimes C)
$$

extends canonically over $u$ to a map

$$
\operatorname{Sym}^{2}(\hat{T}) \stackrel{d}{\rightarrow} \operatorname{Pic}(\check{T} \otimes C) .
$$

It is easy to check the following.

Lemma 10.14. The group $\operatorname{Sym}^{2}(\hat{T})$ is isomorphic to the group of symmetric bilinear maps $J: \check{T} \times \check{T} \rightarrow \mathbb{Z}$.

It follows that the symmetric bilinear map $I=I_{(1, L G)}$ gives an element of $\operatorname{Sym}^{2}(\hat{T})$, and so a divisor class $d(I)$ in $\operatorname{Pic}(\check{T} \otimes C)$, functorially in the elliptic curve $C$. 
In the case that $C$ is the elliptic curve $\mathbb{C}^{\times} / q^{\mathbb{Z}}$ for $q \in D^{\times}(\mathbb{C})$, Looijenga shows that the class of the line bundle $\mathcal{L}(G)^{g}$ is $d(J)$ where $J$ is the bilinear map

$$
J=\frac{1}{2} \sum_{\alpha \in R_{+}} \alpha \otimes \alpha
$$

In fact, his methods can be used to prove the following.

Proposition 10.15. The class in $\operatorname{Pic}(\check{T} \otimes C)$ of $\mathcal{L}(G)$ is $d(I)$.

Proof. Looijenga shows that, in the transcendental theory [Mum70], the image of the Chern class map

$$
\operatorname{Pic}(\check{T} \otimes C) \stackrel{c_{1}}{\longrightarrow} H^{2}(\check{T} \otimes C ; \mathbb{Z})
$$

is

$$
\operatorname{Sym}^{2}(\hat{T}) \cong \operatorname{Sym}^{2}(\hat{T}) \otimes H^{2}(C ; \mathbb{Z}) .
$$

Moreover, using the fact that $c_{1}(\mathcal{O}(-e))$ generates $H^{2}(C ; \mathbb{Z})$, it is easy to check that under this identification we have

$$
c_{1}\left((\alpha \otimes C)^{*} \mathcal{O}(-e)\right)=\alpha \otimes \alpha .
$$

The universal property of $\mathrm{Sym}^{2}$ implies that

$$
\operatorname{Sym}^{2}(\hat{T}) \stackrel{d}{\rightarrow} \operatorname{Pic}(\check{T} \otimes C) \stackrel{c_{1}}{\longrightarrow} \operatorname{Sym}^{2}(\hat{T})
$$

is the identity. It is also easy to check that $c_{1}(\mathcal{L}(G))=I$, so

$$
c_{1}(\mathcal{L}(G))=I=c_{1}(d(I)) .
$$

The proof is completed by the following lemma.

Lemma 10.16. Let $K$ be a non-archimedean field or $\mathbb{C}$; let $q \in D^{\times}(K)$; and let $C$ be the elliptic curve $K^{\times} / q^{\mathbb{Z}}$. Let $I: \check{T} \times \check{T} \rightarrow \mathbb{Z}$ be a $W$-invariant, non-degenerate, symmetric bilinear map. If $\phi$ and $\psi$ are two $W$-invariant pointed maps of degree 2 from $\check{T}$ to $\mathbb{Z}$ such that

$$
\begin{aligned}
& \phi(u+v)=\phi(u)+\phi(v)+I(u, v), \\
& \psi(u+v)=\psi(u)+\psi(v)+I(u, v),
\end{aligned}
$$

then the line bundles $\mathcal{L}(1, I, \phi)$ and $\mathcal{L}(1, I, \psi)$ over $\check{T} \otimes C$ are $W$-equivariantly isomorphic.

Proof. Let $\delta=\phi-\psi$. Then $\delta$ is a $W$-invariant linear map

$$
\check{T} \rightarrow \mathbb{Z} \text {. }
$$

Let $v \in \check{T}$ be the element such that

$$
I(u, v)=\delta(u)
$$

for all $u \in \check{T}$; it is easy to check that $w \cdot v=v$ for $w \in W$. The map

$$
\left(\check{T} \otimes K^{\times}\right) \times K \stackrel{f}{\rightarrow}\left(\check{T} \otimes K^{\times}\right) \times K
$$

given by the formula

$$
f(z, t)=\left(z q^{v}, t\right)
$$

induces the desired isomorphism

$$
\mathcal{L}(1, I, \psi) \underset{\mathcal{L}}{\cong}(1, I, \phi) .
$$




\section{Representations of $L T$}

The description of the representation theory of $L G$ summarized in the previous section depends implicitly on a detailed understanding of the representation theory of $L T$. Although $T$ is neither simple nor simply connected, by the time the dust settles the representation theory of $L T$ is essentially the same.

The group $L T$ possesses a $T$-split central extension

$$
1 \rightarrow \mathbb{T} \rightarrow \tilde{L T} \rightarrow L T \rightarrow 1,
$$

called the "basic" central extension, whose inner product $I=I_{(1, \tilde{L T})}$ induces an isomorphism

$$
\check{T} \underset{I}{\stackrel{I}{\longrightarrow}} \hat{T} .
$$

Indeed, after identifying $\check{T} \cong \mathbb{Z}^{r}$, where $r$ is the rank of $T$, one may take

$$
\begin{aligned}
I(x, y) & =\sum_{i=1}^{r} x_{i} y_{i}, \\
\phi(x) & =\frac{1}{2} \sum_{i=1}^{r} x_{i}\left(x_{i}-1\right) .
\end{aligned}
$$

The representation theory is simpler since now

Proposition 11.2. The affine Weyl group of LT is just

$$
W_{\text {aff }} \cong \check{T} \text {. }
$$

Definition 11.3. A representation of $L T$ of level $k$ is a representation of $L T$ of level $k_{*} L T$ and type 1 .

Once again we simplify the notation.

$$
\begin{aligned}
\operatorname{Rep}_{k}(L T) & =\operatorname{Rep}_{\left(1, k_{*} \tilde{L T}\right)}(L T), \\
\mathcal{L}(T) & =\mathcal{L}(1, \tilde{L T}), \\
\Gamma(T)_{k} & =\Gamma\left(1, k_{*} \tilde{L T}\right)
\end{aligned}
$$

For $k \in \mathbb{Z}, \Gamma(T)_{k}$ is the group of functions

$$
f \in(\mathbb{Z} \llbracket \hat{T} \rrbracket)((q))
$$

which satisfy

$$
f\left(z q^{n}, q\right)=q^{-k \phi(n)} z^{-k I(n)} f(z, q)
$$

for $n \in \check{T}$.

For $k \geq 1$ and $v \in \mathbb{Z}$, let $g_{v, k}$ be the function

$$
g_{v, k}(z, q) \stackrel{\text { def }}{=} \sum_{n \in \check{T}} q^{k \phi(n)+\langle v, n\rangle} z^{k I(n)+v} .
$$

Proposition A.9 gives

Proposition 11.4. If $S$ is a set of coset representatives for $\hat{T} / k \check{T}$, then the functions $g_{v, k}$ for $v \in S$ are a basis for $\Gamma(T)_{k}$. 
Recall (10.6) that

$$
\Phi(q)=\prod_{n \geq 1}\left(1-q^{n}\right) .
$$

Kac Character Formula 11.5. Let $T$ be a torus of rank $r$. The irreducible representations of $L T$ of level $k$ are parametrized by $\hat{T} / k \check{T}$. The character of the irreducible representation corresponding to $v$ is

$$
\chi_{v, k}(t, z, q)=t^{k} \Phi(q)^{-r} g_{v, k}(z, q)
$$

Proof. See sections 9.5 and 14.3 in [PS86].

Theorem 11.6. The character map induces an isomorphism

$$
\operatorname{Rep}_{k}(L T) \cong \Gamma(T)_{k} .
$$

If $F$ is a non-archimedean field and $q$ is a unit of $F$ with $|q|<1$, then this in turn induces an isomorphism

$$
F \underset{\mathbb{Z}((q))}{\otimes} \operatorname{Rep}_{k}(L T) \cong \Gamma \mathbf{E}_{T}\left(p t, \mathcal{L}(T)^{k}\right)(q) .
$$

Remark 11.7. The representation theory of the Heisenberg group provides a direct connection between $\mathbf{E}_{\mathbb{T}}$ and $\tilde{L T}$. In addition to section 9.5 in [PS86] the reader may wish to consult Mum91].

11.1. Calculations for $L \mathbb{T}$. The loop group $L \mathbb{T}$ has only one irreducible representation of level 1, called the "basic representation", corresponding to

$$
0 \in \hat{\mathbb{T}} / \check{\mathbb{T}} \text {. }
$$

In addition to the formula

$$
\chi_{\mathcal{H}}(z, q)=\Phi(q)^{-1} \sum_{k \in \mathbb{Z}} q^{\frac{1}{2} k(k-1)} z^{k}
$$

provided by Theorem 11.5 its character has a well known product expansion which may be obtained from the following construction of the representation.

Let $H$ denote the Hilbert space $L^{2}\left(S^{1}, \mathbb{C}\right)$. It carries an action of $L \mathbb{T}$, but it is not of positive energy. However, $H$ is polarized

$$
H \cong H_{+} \oplus H_{-}
$$

according to the action of $\mathbb{T}_{\text {rot }}$, and the basic representation is a completion of the total exterior power

$$
\mathcal{H}=\hat{\Lambda}\left(H_{+} \oplus \bar{H}_{-}\right)
$$

Since the character of $H_{+} \oplus H_{-}$is

$$
\chi_{H_{+} \oplus \bar{H}_{-}}(z, q)=\sum_{k \geq 0} q^{k} z+\sum_{k>0} q^{k} z^{-1},
$$

the character of $\mathcal{H}$ is

$$
\chi_{\mathcal{H}}(z, q)=(1+z) \prod_{k>0}\left(1+q^{k} z\right)\left(1+q^{k} z^{-1}\right) .
$$

The Tate curve has a point of order 2 , namely the point -1 in $K^{\times} / q^{\mathbb{Z}}$; its preimage in $K^{\times}$is the set of points $-q^{\mathbb{Z}}$. The formula (11.10) shows that $\chi_{\mathcal{H}}(z, q)$, considered as a function on $K$, vanishes to first order at these points $-q^{\mathbb{Z}}$. It follows 
that the line bundle $\mathcal{L}(\mathbb{T})$ is the line bundle defined by the divisor $(-1)$ on Tate, and indeed one has the equation

$$
\chi_{\mathcal{H}}(z, q)=\theta(-z, q)
$$

with $\theta$ given by (2.8). This observation was one starting point of our investigation.

\section{Part III. Power operations and loop groups}

\section{ISOGENIES AND THEIR EFFECT ON THETA FUNCTIONS}

In section 9.1 , we showed that a $G$-split central extension $E$ of a loop group $L G$ determines a $W$-equivariant line bundle $\mathcal{L}(n, E)$ over $\mathbf{E}_{T}\left(q^{n}\right)$, and that the character of a representation of level $E$ and type $n$ is naturally a $W$-invariant section of $\mathcal{L}(n, E)$.

In section 6.3, we studied two basic families of isogenies of the Tate curve, namely

$$
\begin{array}{ll}
g_{1, b}: \operatorname{Tate}(q) \rightarrow \operatorname{Tate}(r) & \text { over } D(1, b), \\
g_{a, 1}: \operatorname{Tate}(q) \rightarrow \operatorname{Tate}\left(q^{a}\right) & \text { over } D(a, 1)=\mathbb{Z}((q)) .
\end{array}
$$

The notation Tate $\left(q^{a}\right)$ makes implicit use of the specialization map

$$
\begin{aligned}
\mathbb{Z}((q)) \stackrel{s_{a}}{\longrightarrow} \mathbb{Z}((q)) \\
q \mapsto q^{a}
\end{aligned}
$$

as $\operatorname{Tate}\left(q^{a}\right) \stackrel{\text { def }}{=} s_{a}^{*}$ Tate. For $\zeta^{b}=1$ it is convenient also to consider the specialization

$$
\begin{aligned}
D(1, b) & \stackrel{s_{b, \zeta}}{\longrightarrow} \mathbb{Z}[\zeta]((q)) \\
& \cong q^{b} \\
r & \mapsto \zeta q,
\end{aligned}
$$

under which $g_{1, b}$ becomes an isogeny

$$
g_{1, b, \zeta}: \operatorname{Tate}\left(q^{b}\right) \rightarrow \operatorname{Tate}(\zeta q) .
$$

If $I: \check{T} \times \check{T} \rightarrow \mathbb{Z}$ and $\phi: \check{T} \rightarrow \mathbb{Z}$ are as in section 9.1 satisfying

$$
\phi(u+v)=\phi(u)+\phi(v)+I(u, v),
$$

then it is easy to check the following.

Lemma 12.1.

$$
\begin{aligned}
g_{1, b, \zeta}^{*} \mathcal{L}(1, I, \phi) & \cong \mathcal{L}(b, b I, \phi(b-)), \\
g_{a, 1}^{*} \mathcal{L}(a, I, \phi) & \cong \mathcal{L}(1, a I, \phi), \\
s_{a}^{*} \mathcal{L}(1, I, \phi) & \cong \mathcal{L}(a, I, a \phi), \\
g_{a, 1}^{*} s_{a}^{*} \mathcal{L}(1, I, \phi) & \cong \mathcal{L}(1, a I, a \phi) \cong \mathcal{L}(1, I, \phi)^{a} .
\end{aligned}
$$

If $f$ is a section of the line bundle $\mathcal{L}(1, I, \phi)$, viewed as a $\theta$ function $f(z, q)$, then

$$
\begin{aligned}
g_{1, b, \zeta}^{*} s_{b, \zeta}^{*} f(z, q) & =f(z, \zeta q), \\
s_{a}^{*} f(z, q) & =f\left(z, q^{a}\right) .
\end{aligned}
$$

If $f$ is a section of $\mathcal{L}(a, I, \phi)$, then

$$
g_{a, 1}^{*} f(z, q)=f\left(z^{a}, q\right) .
$$


If $f$ is a section of $\mathcal{L}(1, I, \phi)$, then

$$
g_{a, 1}^{*} s_{a}^{*} f(z, q)=f\left(z^{a}, q^{a}\right) .
$$

12.1. Isogenies and representations. The isogenies of the Tate curve are related to a family of homomorphisms

$$
f_{a, b}: L G \rtimes a b \stackrel{[b] \rtimes \sigma_{a}}{\longrightarrow} L G \rtimes 1
$$

generated by

$$
\begin{aligned}
\sigma_{a}(\gamma, \lambda) & =\left(\gamma, \lambda^{a}\right), \\
{[b] \gamma(z) } & =\gamma\left(z^{b}\right) .
\end{aligned}
$$

If $E$ is a $G$-split central extension, then there is a homomorphism

$$
E \rtimes a b=[b]^{*} E \rtimes a b \rightarrow E \rtimes 1
$$

covering $f_{a, b}$. It is easy to check the following.

Lemma 12.4. If $I=I_{(1, E)}$ and $\phi=\phi_{(1, E)}$, then

$$
\begin{aligned}
I_{\left(a b,[b]^{*} E\right)} & =a b I, \\
\phi_{\left(a b,[b]^{*} E\right)}(-) & =a \phi(b-) .
\end{aligned}
$$

Moreover, if $V$ is a representation of level $E$ and type 1, then

$$
\begin{aligned}
\mathcal{L}(a, E) & \cong s_{a}^{*} \mathcal{L}(1, E), \\
\mathcal{L}\left(b,[b]^{*} E\right) & \cong g_{1, b}^{*} s_{b, 1}^{*} \mathcal{L}(1, E) .
\end{aligned}
$$

If $V$ is a representation of $L G$ of level $E$ and type $c$, then

$$
\begin{aligned}
\chi_{\sigma_{a}^{*} V} & =s_{a}^{*} \chi_{V}, \\
\chi_{[b] * V} & =g_{1, b}^{*} \chi_{V} .
\end{aligned}
$$

Comparing Lemma 12.4 with Lemma 12.1 we have

Proposition 12.5. (i) The pull-back of characters along the magnification of loops [b] corresponds to the pull-back of sections of the line bundle $\mathcal{L}(1, E)$ along the isogeny $g_{1, b, 1}$.

(ii) The pull-back of characters along the characters along the homomorphism $\sigma_{a}$ corresponds to the specialization of sections of the line bundle $\mathcal{L}(1, E)$ under the map $s_{a}(q)=q^{a}$.

There are two families of isogenies missing from Proposition 12.5; the isogeny

$$
\operatorname{Tate}(q) \stackrel{g_{a, 1}}{\longrightarrow} \operatorname{Tate}\left(q^{a}\right)
$$

and the isogenies

$$
\operatorname{Tate}\left(q^{b}\right) \stackrel{g_{1, b, \zeta}}{\longrightarrow} \operatorname{Tate}(\zeta q) .
$$

We deal with $g_{1, b, \zeta}$ in the next section, and turn to $g_{a, 1}$. 
12.2. The Adams operation. From the formula

$$
g_{a, 1}^{*} s_{a}^{*} f(z, q)=f\left(z^{a}, q^{a}\right)
$$

of Lemma 12.1 it is clear that the isogeny $g_{a, 1}$ should correspond to the Adams operation. We simply observe that such an operation can be defined. If $V$ and $W$ are two representations of $L G$ of type 1 and level $E$ and $F$ respectively, then there is a tensor product of $V$ and $W$ : by assumption the space

$$
V \hat{\otimes} W(k) \stackrel{\text { def }}{=} \bigoplus_{i+j=k} V(i) \otimes W(j)
$$

is finite dimensional, and zero for $k$ sufficiently small. There is a natural inner product on

$$
\bigoplus_{k} V \hat{\otimes} W(k),
$$

and we may define $V \hat{\otimes} W$ to be the Hilbert space completion of (12.6) with respect to this inner product. It is a representation of $L G$ of level $E \times F$.

In particular, if $V$ is a representation of level $E$, then $V^{\hat{\otimes} a}$ is a representation of level $a_{*} E$; in fact it is a representation of $\Sigma_{a} \times a_{*} E$. As such it may be decomposed as a representation of $\Sigma_{a}$; the usual procedure of evaluating on the class of an $a$-cycle yields a map

$$
\operatorname{Rep}_{(1, E)}(L G) \stackrel{\Psi^{a}}{\longrightarrow} \operatorname{Rep}_{\left(1, a_{*} E\right)} .
$$

The usual argument shows that the effect on characters is given by the formula

$$
\chi_{\Psi^{a} V}(z, q)=\chi_{V}\left(z^{a}, q^{a}\right)=g_{a, 1}^{*} s_{a}^{*} \chi_{V} .
$$

Proposition 12.7. The Adams operation $\Psi^{a}$ on characters of representations corresponds to the operation $\Psi^{a, 1}$ of section 6.3; namely,

$$
\chi_{\Psi^{a} V}=g_{a, 1}^{*} s_{a}^{*} \chi_{V} .
$$

\section{Finite subgroups of the Rotation GRoup AND MAGNIFICATION OF LOOPS}

13.1. Representations of type $b$. A key point about representations of type $b$ is

Lemma 13.1. The subgroup of $\mathbb{T}_{\text {rot }}[b]$ of b-order torsion is contained in the center of $E \rtimes b$.

Thus a representation of level $E$ and type $b$ may be decomposed as a representation of $\mathbb{T}_{\text {rot }}[b]$. After a choice of map

$$
\begin{aligned}
& \mathbb{T}_{\text {rot }}[b]^{*} \rightarrow \mu[b] \\
&\left.q\right|_{\mathbb{T}_{\text {rot }}[b]} \mapsto \zeta,
\end{aligned}
$$

we may write the character of $V$ as a representation of $\mathbb{T}_{\text {rot }}[b] \times E$ as

$$
\chi_{V}(z, q, \zeta)=\chi_{V}(z, q \zeta)=\sum_{k \gg-\infty} q^{k} \zeta^{k} p_{k}
$$

with $p_{k} \in \mathbb{Z}[\hat{T}]$. 
13.2. Magnification of loops. In particular, if $V$ is a representation of $L G$ of level $E$ and type 1 , then $[b]^{*} V$ is a representation of level $[b]^{*} E$ and type $b$. Choose a map

$$
\mathbb{T}_{\text {rot }}[b]^{*} \rightarrow \mu[b]
$$

let us name the map by the image of

$$
\left.q\right|_{\mathbb{T}_{\text {rot }}[b]} \mapsto \zeta .
$$

Then the character of $f_{1, b}^{*} V$ as a representation of $\mathbb{T}_{\text {rot }}[b] \times[b]^{*} E$ is

$$
\chi_{f_{1, b}^{*} V}(z, q, \zeta)=\chi_{V}(z, q \zeta)
$$

it is a section of $\mathcal{L}(b, b I, \phi(b-))$.

Proposition 13.3. The process of pulling back a representation along $f_{1, b}$ and then viewing it as a character of $\mathbb{T}_{r o t}[b] \times[b]^{*} E$ using (13.2) corresponds to the operation $\Psi^{1, b, \zeta}$ of section [6.3; namely,

$$
\chi_{f 1, b^{*} V}(z, q, \zeta)=g_{1, b, \zeta}^{*} s_{b, \zeta}^{*} \chi_{V} .
$$

Remark 13.4. According to Lemma 12.4 $I_{\left(b,[b]^{*} E\right)}=b I$. In the case that $G$ is simple and simply connected, it follows that the central extension $[b]^{*} \tilde{L G}$ is canonically isomorphic to $b_{*} \tilde{L} G$, so $[b]^{*} V$ is a representation of $L G$ of "level $b$ " in the sense of Definition 10.1. In fact, this is also the case when $\tilde{L G}$ is the "basic" central extension of a simply-laced group [PS86, §9].

\subsection{Decomposition of representations of type $b$. If}

$$
E \rtimes b \stackrel{\rho}{\rightarrow} \operatorname{Aut}(V)
$$

is a representation of type $b$, let $\rho_{j}: E \rightarrow U\left(V_{j}\right)$ be the the summand on which $\mathbb{T}_{\text {rot }}[b]$ acts by the character $\left.q^{j}\right|_{\mathbb{T}_{\text {rot }}[b]}$.

Proposition 13.5. The representation $q^{-j} \otimes \rho_{j}$ factors through

$$
E \rtimes b \stackrel{\sigma_{b}}{\longrightarrow} E \rtimes 1 .
$$

Let $\rho_{(j)}$ or simply $V_{(j)}$ denote the resulting representation. If

$$
\chi_{V}=\sum_{k \gg-\infty} q^{k} p_{k}
$$

with $p_{k} \in \mathbb{Z}[\hat{T}]$, then

$$
\chi_{V_{(j)}}=\sum_{k \gg-\infty} q^{k} p_{b k+j}
$$

\section{Appendix A. Theta functions}

The theta functions in section 10 are equivalent to those in [Kac85] and Loo76], but our notation is different from theirs. To aid the reader with the translation, we give a proof (along the lines of [Loo76]) of Proposition 10.2, which applies as well to Proposition 11.4. 


\section{A.1. Notation.}

$$
\begin{aligned}
L:= & \text { a free abelian group of rank } r, \\
\phi:= & \text { a non-negative map of degree } 2 \\
& L \rightarrow \mathbb{Z}, \\
I:= & \text { a non-degenerate symmetric bilinear form } \\
& L \times L \rightarrow \mathbb{Z}, \text { such that } \\
& \phi(u+v)=\phi(u)+\phi(v)+I(u, v), \\
\mathbf{K}:= & L \otimes \mathbb{G}_{m}, \\
\mathbf{E}:= & L \underset{\mathbb{Z}}{\otimes} \text { Tate, } \\
q^{L}:= & L \underset{\mathbb{Z}}{\otimes} \\
= & \left\{n \otimes q^{k} \mid n \in L ; k \in \mathbb{Z}\right\} \subset \mathbf{K}, \\
M:= & \text { Hom }[L, \mathbb{Z}], \\
\langle-,-\rangle & \text { the evaluation } L \times M \rightarrow \mathbb{Z} .
\end{aligned}
$$

The situation of the rest of the paper can be recovered by taking $L=\check{T}$. In section 10] we have

$$
\phi(\ell)=\frac{1}{2} I(\ell, \ell) .
$$

In the situation of section 11, we have

$$
\phi(\ell)=\frac{1}{2} \sum_{i=1}^{r} \ell_{i}\left(\ell_{i}-1\right)
$$

in terms of an isomorphism $\check{T} \cong \mathbb{Z}^{r}$.

As usual, we denote by

$$
\prod z_{i}^{n_{i}}
$$

the element of $\mathbf{K}$ which might also be written $\sum n_{i} \otimes z_{i}$.

The adjoint of $I$ is an injection

$$
L \stackrel{I}{\longrightarrow} M
$$

which we also denote by $I$. For example, if $n \in L$, then we obtain a homomorphism

$$
L \otimes \mathbb{G}_{m} \stackrel{I(n)}{\longrightarrow} \mathbb{G}_{m}
$$

for $z=\prod z_{i}^{n_{i}}$ we have

$$
\begin{aligned}
z^{I(n)} & =I(n)(z) \\
& =I(n)\left(\prod z_{i}^{n_{i}}\right) \\
& =\prod z_{i}^{I\left(n, n_{i}\right)} .
\end{aligned}
$$

The behavior of $\phi$ and $I$ is quadratic in the following sense.

Lemma A.2. Suppose that $k$ is an integer greater than 0 . For each $N \in \mathbb{Z}$ and $v \in M$, the set

$$
\{\ell \in L \mid\langle\ell, v\rangle+k \phi(\ell)-k I(\ell, \ell) \geq N\}
$$

is finite. 
Proof. This is standard. Let $e_{1}, \ldots, e_{r}$ be a basis for $L$, and define rational constants $a_{i}, b_{i j}, c_{i}$, and $v_{i}$ by the formulae

$$
\begin{aligned}
a_{i} & =\frac{k}{2} I\left(e_{i}, e_{i}\right), \\
b_{i j} & =k I\left(e_{i}, e_{j}\right), \\
c_{i} & =k \phi\left(e_{i}\right), \\
v_{i} & =\left\langle e_{i}, v\right\rangle .
\end{aligned}
$$

Since $I$ is positive definite, the $a_{i}$ are strictly positive. If $\ell=\sum \ell_{i} e_{i}$, then it is easy to check that

$$
\langle\ell, v\rangle+k \phi(\ell)-k I(\ell, \ell)=\sum_{i=1}^{r}\left(v_{i}-a_{i}+c_{i}\right) \ell_{i}-\sum_{1 \leq i<j \leq r} b_{i j} \ell_{i} \ell_{j}-\sum_{i} a_{i} \ell_{i}^{2} .
$$

The set of $\ell$ for which this quadratic polynomial is positive is finite.

A map of sets

$$
c: \mathbb{Z} \times M \rightarrow \mathbb{Z}
$$

will be recorded as a formal sum

$$
f(z, q)=\sum_{\substack{n \in \mathbb{Z} \\ v \in M}} c(n, v) q^{n} z^{v} .
$$

Definition A.3. We denote by $\Gamma(k, I, \Phi)$ or just the $\Gamma_{k}$ group of functions

$$
c: \mathbb{Z} \times M \rightarrow \mathbb{Z}
$$

which satisfy the following:

(i) There is a $b \in \mathbb{Z}$ such that

$$
c(n, v)=0
$$

for all $v \in M$ and $n<b$.

(ii) The formal sum $f$ corresponding to $c$ satisfies the equation

$$
f\left(z q^{\ell}\right)=q^{-k \phi(\ell)} z^{-k I(\ell)} f(z)
$$

for all $\ell \in L$.

Lemma A.5. The equation A.4 is equivalent to

$$
c(n, v-k I(\ell))=c(n+\langle\ell, v\rangle+k \phi(\ell)-k I(\ell, \ell), v)
$$

for $n \in \mathbb{Z}$ and $v \in M$.

Proof. The expansion of the left-hand side of

$$
f\left(z q^{\ell}, q\right)=q^{-k \phi(\ell)} z^{-k I(\ell)} f(z)
$$

is

$$
f\left(z q^{\ell}, q\right)=\sum_{\substack{n \in \mathbb{Z} \\ v \in M}} c(n, v) q^{n+\langle\ell, v\rangle} z^{v} .
$$

The right-hand side is

$$
q^{-k \phi(\ell)} z^{-k I(w)} f(z)=\sum_{\substack{n \in \mathbb{Z} \\ v \in M}} c(n, v) q^{n-k \phi(\ell)} z^{v-k I(\ell)} .
$$


Then, comparing coefficients, we have

$$
\begin{aligned}
c(n, v-k I(\ell)) & =\text { coefficient of } q^{n+\langle\ell, v-k I(\ell)\rangle} z^{v-k I(\ell)} \text { on left } \\
& =\text { coefficient of } q^{n+\langle\ell, v\rangle-k I(\ell, \ell)} z^{v-k I(\ell)} \text { on left } \\
& =c(v, n+\langle\ell, v\rangle+k \phi(\ell)-k I(\ell, \ell)) \text { on right. }
\end{aligned}
$$

\section{Proposition A.6.}

$$
\Gamma_{0}=\mathbb{Z}((q)) .
$$

Proof. First, for each $v$ we know that $c(n, v)=0$ for $n$ sufficiently small. When $k=0$, Lemma A.5 says that

$$
c(n, v)=c(n+\langle\ell, v\rangle, v)
$$

for all $\ell \in L$. Unless $v=0$, it follows that $c(n, v)=0$.

Proposition A.7. For $k \geq 0$, if $f \in \Gamma_{k}$, then $f$ is an element of the ring

$$
(\mathbb{Z}[M])((q)) \text {. }
$$

Proof. Write

$$
f(z, q)=\sum_{n, v} c(n, v) z^{v} q^{n} .
$$

Let $S$ be a set of coset representatives for $M / k I(L)$. It suffices to show that for each $n \in \mathbb{Z}$ and $s \in S$, the set

$$
\{\ell \in L \mid c(n, s-k I(\ell)) \neq 0\}
$$

is finite. Lemma A.5 shows that

$$
c(n, s-k I(w))=c(n+\langle\ell, s\rangle+k \phi(\ell)-k I(\ell, \ell), s) .
$$

Lemma A.2 and the assumption that $f \in \Gamma_{k}$ shows that the coefficient on the right is non-zero for only finitely many $w$.

A choice $S$ of coset representatives for $M / k I(L)$ determines a basis for $\Gamma_{k}$. For $v \in M$, let $g_{v, k}$ be the function

$$
g_{v, k}(z, q)=g_{v, k}(z, q, I) \stackrel{\text { def }}{=} \sum_{n \in L} q^{k \phi(n)+\langle n, v\rangle} z^{k I(n)+v} .
$$

Lemma A.8. $g_{v, k}$ is an element of $\Gamma_{k}$.

Proof. For $\ell \in L$ we have

$$
\begin{aligned}
g_{v, k}\left(z q^{\ell}, q\right) & =\sum_{n \in L} q^{k \phi(n)+\langle n, v\rangle} z^{k I(n)+v} q^{k I(n, \ell)+\langle\ell, v\rangle} . \\
& =q^{-k \phi(\ell)} \sum_{n \in L} q^{k \phi(n+\ell)+\langle n+\ell, v\rangle} z^{k I(n)+v} \\
& =q^{-k \phi(\ell)} z^{-I(\ell)} \sum_{n \in L} q^{k \phi(n+\ell)+\langle n+\ell, v\rangle} z^{k I(n+\ell)+v} \\
& =q^{-k \phi(\ell)} z^{-I(\ell)} g_{v, k}(z, q) .
\end{aligned}
$$


Proposition A.9. If $k>0$ and if $S$ is a set of coset representatives for $M / k L$, then the functions $g_{s}$ for $s \in S$ are a basis for $\Gamma_{k}$ over $\mathbb{Z}((q))$.

Proof. The $g_{s, k}$ for $s \in S$ are certainly linearly independent over $\mathbb{Z}((q))$; just compare coefficients of $q^{k \phi(0)} z^{s}$ for $s \in S$. Now suppose that $f \in \Gamma_{k}$, and write

$$
f(z, q)=\sum_{n, v} c(n, v) z^{v} q^{n} .
$$

Define $a_{s} \in \mathbb{Z}((q))$ by the formula

$$
a_{s}=\sum_{n \in \mathbb{Z}} c(n, s) q^{n} .
$$

Then $\sum_{s \in S} a_{s} g_{s, k}$ and $f$ both live in $\Gamma_{k}$. The coefficient of $z^{s} q^{n}$ in their difference is 0 . By Lemma A.5, they are equal.

Now let $F$ be a complete, non-archimedean field, and let $q \in F$ be an element with $|q|<1$ : we obtain a line bundle $\mathcal{L}(I, \phi)$ over the variety $L \otimes \operatorname{Tate}(q)$.

Proposition A.10. If $k>0$ and if $S$ is a set of coset representatives for $M / k I(L)$, then the functions $g_{s, k}$ for $s \in S$ are an $F$-basis for the global sections of $\mathcal{L}(I, \phi)$.

Proof. In view of Proposition A.9 what remains is to remark that the power series $g_{v, k}(z, q)$ converges since the $|q|<1$ and the coefficient of $q^{N}$ is a Laurent polynomial in $r$ variables of degree on the order of $\sqrt{N}$ in each variable.

\section{REFERENCES}

[Ada71] J. Frank Adams. A variant of E. H. Brown's representability theorem. Topology, 10, 1971. MR 44:1018

[And95] Matthew Ando. Isogenies of formal group laws and power operations in the cohomology theories $E_{n}$. Duke Math. J., 79(2), 1995. MR 97a:55006

[Bak90] Andrew Baker. Hecke operators as operations in elliptic cohomology. J. Pure and Applied Algebra, 63:1-11, 1990. MR 91m:55005

[BMMS86] R. Bruner, J. P. May, J. E. McClure, and M. Steinberger. $H_{\infty}$ ring spectra, volume 1176 of Lecture Notes in Mathematics. Springer, 1986. MR 88e:55001

[Bre83] Lawrence Breen. Fonctions thêta et théorème du cube, volume 980 of Lecture Notes in Mathematics. Springer, 1983. MR 87d:14036

[Bry90] Jean-Luc Brylinski. Representations of loop groups, Dirac operators on loop space, and modular forms. Topology, 29:461-480, 1990. MR 91:58151

[BW89] A. J. Baker and U. Würgler. Liftings of formal groups and the Artinian completion of $v_{n}^{-1}$ BP. Math. Proc. Cambridge Phil. Soc., 106:511-530, 1989. MR 90i:55008

[Del75] Pierre Deligne. Courbes elliptiques: formulaire (d'apres J. Tate). In Modular functions of one variable IV, volume 476 of Springer Lecture Notes, 1975. MR 52:8135

[DG70] Michel Demazure and Pierre Gabriel. Groupes algébriques, tome I. North-Holland, 1970. MR 46:1800

[DR73] P. Deligne and M. Rapoport. Les schemas de modules de courbes elliptiques. In Modular functions of one variable II, volume 349 of Springer Lecture Notes, 1973. MR 52:3177

[Dri73] V. G. Drinfeld. Elliptic modules. Math. USSR, Sbornik, 23, 1973. MR 52:5580

[Fre82] I. B. Frenkel. Representations of affine Lie algebras, Hecke modular forms, and Korteweg-de Vries type equations. In Lie algebras and related topics, volume 933 of Lecture Notes in Mathematics. Springer, 1982. MR 84f:17006

[GKV95] V. Ginzburg, M. Kapranov, and E. Vasserot. Elliptic algebras and equivariant elliptic cohomology, 1995. Preprint.

[Gro94] Ian Grojnowski. Delocalized equivariant elliptic cohomology, 1994. Submitted to MRL.

[HH95] Michael J. Hopkins and John R. Hunton. On the structure of spaces representing a Landweber exact cohomology theory. Topology, 34, 1995. MR 95k:55009 
[HKR92] Michael J. Hopkins, Nicholas J. Kuhn, and Douglas C. Ravenel. Generalized groups, characters and complex oriented cohomology theories. Preprint, 1992.

[HS97] Mark Hovey and Neil P. Strickland Morava K-theories and localization, Mem. Amer. Math. Soc., 1997. MR 99b:55017

[HT98] John Hunton and Paul Turner. The homology of spaces representing exact pairs of homotopy functors, 1998. Preprint.

[Igu59] J. Igusa. On the transformation theory of elliptic functions. Amer. J. Math., 81, 1959. MR 21:3421

[JW73] David C. Johnson and W. Stephen Wilson. Projective dimension and Brown-Peterson homology. Topology, 12:327-353, 1973. MR 48:12596

[Kac85] Victor G. Kac. Infinite dimensional Lie algebras. Cambridge University Press, second edition, 1985. MR 87c:17023

[Kat81] Nicholas M. Katz. Serre-Tate local moduli. In Surfaces algébriques: séminaire de géométrie algébrique d'Orsay 1976-1978, volume 868 of Lecture Notes in Mathematics, pages 138-202. Springer, 1981. MR 83k:14039b

[KM85] Nicholas M. Katz and Barry Mazur. Arithmetic moduli of elliptic curves, volume 108 of Annals of Math. Studies. Princeton University Press, 1985. MR 86i:11024

[KW90] V. G. Kac and M. Wakimoto. Branching functions for winding subalgebras and tensor products. Acta Appl. Math. 21, 1990. MR 92a:17037

[Loo76] Eduard Looijenga. Root systems and elliptic curves. Inventiones Math., 38, 1976. MR 57:6015

[LRS95] Peter S. Landweber, Douglas C. Ravenel, and Robert E. Stong. Periodic cohomology theories defined by elliptic curves. Contemporary Mathematics, 181, 1995. MR 96i:55009

[LT65] Jonathan Lubin and John Tate. Formal complex multiplication in local fields. Annals of Math., 81:380-387, 1965. [MR 30:3094]

[Lub67] Jonathan Lubin. Finite subgroups and isogenies of one-parameter formal Lie groups. Annals of Math., 85:296-302, 1967. MR 35:189

[Mil89] Haynes Miller. The elliptic character and the Witten genus. In Algebraic topology (Mark Mahowald and Stewart Priddy, editors), Northwestern University, 1988, volume 96 of Contemporary Math. Amer. Math. Soc., 1989. MR 90i:55005

[Mor89] Jack Morava. Forms of K-theory. Math. Z., 201:401-428, 1989. MR 90e:55011

[Mum70] David Mumford. Abelian Varieties, volume 5 of Tata Institute of Fundamental Research Series in Mathematics. Oxford University Press, 1970. MR 44:219

[Mum91] David Mumford. Tata lectures on theta III, volume 97 of Progress in Mathematics. Birkhäuser, 1991. MR 93d:14065

[PS86] Andrew Pressley and Graeme Segal. Loop groups. Oxford Univ. Press, 1986. MR 88i:22049

[RW77] Douglas C. Ravenel and W. Stephen Wilson. The Hopf ring for complex cobordism. J. Pure and Applied Algebra, 9, 1977. MR 56:6644

[Seg88] Graeme Segal. Elliptic cohomology. Seminaire Bourbaki, (695), 1988. MR 91b:55005

[Sil86] Joseph Silverman. The arithmetic of elliptic curves, volume 106 of Graduate Texts in Mathematics. Springer, 1986. MR 87g:11070

[Sil94] Joseph Silverman. Advanced topics in the arithmetic of elliptic curves, volume 151 of Graduate Texts in Mathematics. Springer, 1994. MR 96b:11074

[Str97] Neil P. Strickland. Finite subgroups of formal groups. J. Pure and Applied Algebra, 121:161-208, 1997. MR 98k:14065

[Str98] Neil P. Strickland. Formal schemes and formal groups, 1998. Preprint.

[Wil82] W. Stephen Wilson. Brown-Peterson homology: an introduction and sampler, volume 48 of Regional Conference Series in Math. American Math. Society, 1982. MR 83j: 12576

Department of Mathematics, The University of Virginia, Charlottesville, Virginia

Current address: Department of Mathematics, The University of Illinois at Urbana-Champaign, 1409 W. Green St., Urbana, Illinois 61801

E-mail address: ando@math.jhu.edu 